\title{
Quantum-Spacetime Scenarios and Soft Spectral Lags of the Remarkable GRB130427A
}

\author{
Giovanni Amelino-Camelia, ${ }^{1}$ Fabrizio Fiore, ${ }^{2}$ Dafne Guetta, ${ }^{2,3}$ and Simonetta Puccetti ${ }^{2,4}$ \\ ${ }^{1}$ Dipartimento di Fisica, Sapienza Università di Roma and INFN, Sez. Roma1, P.le A. Moro 2, 00185 Roma, Italy \\ ${ }^{2}$ INAF-Osservatorio Astronomico di Roma, Via Frascati 33, 00040 Monteporzio Catone, Italy \\ ${ }^{3}$ Department of Physics, ORT Braude, Snunit 51 Street P.O. Box 78, 21982 Karmiel, Israel \\ ${ }^{4}$ ASI Science Data Center, Via Galileo Galilei, 00044 Frascati, Italy \\ Correspondence should be addressed to Giovanni Amelino-Camelia; amelino@romal.infn.it
}

Received 2 October 2013; Accepted 25 November 2013; Published 20 January 2014

Academic Editor: Piero Nicolini

Copyright (c) 2014 Giovanni Amelino-Camelia et al. This is an open access article distributed under the Creative Commons Attribution License, which permits unrestricted use, distribution, and reproduction in any medium, provided the original work is properly cited. The publication of this article was funded by SCOAP ${ }^{3}$.

\begin{abstract}
We process the Fermi LAT data on GRB130427A using the Fermi Science Tools, and we summarize some of the key facts that render this observation truly remarkable. We then perform a search of spectral lags, of the type that has been of interest for its relevance in quantum-spacetime research. We do find some evidence of systematic soft spectral lags: when confining the analysis to photons of energies greater than $5 \mathrm{GeV}$ there is an early hard development of minibursts within the burst. The effect is well characterized by a linear dependence, within such a miniburst, of the detection time on energy. We also observe that some support for these features is noticeable also in earlier Fermi-LAT GRBs. Some aspects of the comparison of these features for GRBs at different redshifts could be described within a quantum-spacetime picture, but taking into account results previously obtained by other studies we favour the interpretation as intrinsic properties of GRBs. Even if our spectral lags do turn out to have astrophysical origin their understanding will be important for quantum-spacetime research, since any attempt to reveal minute quantum-spacetime-induced spectral lags evidently requires a good understanding of intrinsic mechanisms at the sources that can produce spectral lags.
\end{abstract}

\section{Introduction and Motivation}

The study of Gamma-Ray Bursts (GRBs) has been for some time one of the main themes of astrophysics research, and over the last 15 years it became also of interest for research on quantum gravity. These more recent developments look at GRBs as signals and hope to decode properties of quantumspacetime from the implications of spacetime quantization for how such signals propagate from the distant source to our telescope. Even tiny quantum-spacetime effects modifying the structure of the signal could cumulate along the way, as the signal travels over cosmological distances.

As we here contribute to assess, the remarkable very recent observation of GRB130427A is bound to teach us a lot about the astrophysics of GRBs and has the potential to also empower some of the quantum-spacetime studies of GRBs.

This GRB130427A was extremely powerful, also thanks to the fact that it was among the nearest long GRBs observed. We here take the perspective that GRB130427A is an opportunity to look at a long GRB in "high resolution," allowing us to notice features which could not be noticed in previous GRBs.

In this study we test the potentialities of using GRB130427A in this way by focusing on a much studied class of effects, involving spectral lags with a linear dependence on energy. These possible effects deserve intrinsic interest and their study is further motivated by quantum-spacetime research: in some quantum-spacetime pictures one has that a group of photons ideally emitted all in exact simultaneity at some distant source should reach our telescope with systematic linear spectral lags.

In the next section, besides discussing some aspects of the exceptionality of GRB130427A and the procedures we followed for retrieving Fermi-LAT data on this GRB, we briefly describe the quantum-spacetime picture that inspired this GRB phenomenology. 
Then in Section 3 we look at GRB130427A Fermi-LAT data from the perspective of a search of linear spectral lags. We do not find any evidence of such spectral lags on the low side of the LAT energy range, but we notice that if one restricts attention to GRB130427A photons with energy in excess of $5 \mathrm{GeV}$ there is some rather robust evidence of spectral lags, specifically linear soft spectral lags (lags that are linear in energy and such that higher-energy photons are detected earlier). This feature is even more pronounced if one restricts attention to GRB130427A photons with energy in excess of $15 \mathrm{GeV}$. In the course of characterizing these spectral lags we also stumble upon a feature which might be labeled "hard minibursts": if one restricts attention to GRB130427A photons with energy in excess of $5 \mathrm{GeV}$ a significant fraction of them is found to be part of bursts that last much shorter than the time scale set by the overall duration of the GRB130427A signal in the LAT. Our analysis finds that the first photon in such hard minibursts is always the highest-energy photon in the miniburst.

Section 4 is where we try to use our findings for GRB130427A as guidance for noticing features in data available on previous GRBs.

In the closing Section 5 we offer a perspective on our findings and comment on various scenarios for their interpretation.

\section{Preliminaries on GRB130427A and Quantum-Spacetime}

2.1. The Remarkable GRB130427A and Fermi-LAT Data. At 07:47:06 UT on 27 April, 2013, Fermi-LAT detected [1] high-energy emission from GRB 130427A, which was also detected [2] at lower energies by Fermi-GBM and by several other telescopes including Swift [3], Konus-Wind [4], SPIACS/INTEGRAL [5], and AGILE [6].

From these early (and however sketchy) reports it emerges that GRB130427A is a record setter in many ways. From the point of view of high-energy astrophysics and fundamental physics, relevant for the study we are here reporting, particularly significant is the fact that GRB130427A is the new record holder as the highest-fluence LAT-detected GRB. Moreover the GRB130427A signal contains $a \simeq 95 \mathrm{GeV}$ photon, which establishes the new record for a GRB, by a very wide margin (all previous Fermi-LAT GRB-photons had energy lower than $35 \mathrm{GeV}$ ).

Indications of an extreme fluence for this GRB is also confirmed at lower energies by the SPI-ACS detector onboard INTEGRAL and by Swift, that reported an extremely luminous X-ray afterglow.

Looking back at GRB catalogues one can tentatively estimate that such an exceptional GRB is only observed not more frequently than once in a quarter century (a good benchmark [7], on the basis of fluence, might be GRB881024), and the opportunity is exciting since the quality of our telescopes made so much progress over this last quarter of a century.

The long list of records is surely in part due to the fact that GRB130427A was a rather near long GRB. The redshift was determined [8-10] to be $0.3399 \pm 0.0002$, so we probably got to see from up close (in "high definition") a GRB which already intrinsically was rather powerful.

For the purposes of the analysis we are here reporting, we retrieved LAT Extended Data, in a circular region centered on the optical position [11] R.A. $=11: 32: 32.84$, Dec. $=+27: 41: 56$ from the Fermi LAT data server.

We prepared the data files for the analysis using the LAT ScienceTools-v9r31p1 package, which is available from the Fermi Science Support Center. An overall characterization of the data we retrieved is offered in Figure 1, which shows the Fermi-LAT light curve of GRB130427A.

Following the photon selection suggested by the Fermi team for GRB analysis, we selected all the events of "P7TRANSIENT" class or better, in the energy range $100 \mathrm{MeV}-300 \mathrm{GeV}$ and with the zenith angle $\leq 100 \mathrm{deg}$. "P7TRANSIENT" class contains lower quality photons, the "transient" class events. These cuts are optimized [12] for transient sources for which the relevant timescales are sufficiently short that the additional residual charged-particle backgrounds are much less significant. We selected photons in a circular region centered on the GRB130427A optical position and with a maximum radius of 10 degrees which ensures that all GRB photons ("FRONT" and "BACK," as described in [13]) are considered. Nevertheless, since our study is mainly focused on photons with energy greater than $5 \mathrm{GeV}$ and because the LAT Point Spread Function is energy dependent, we have decided to use also a more conservative source extraction region with a maximum radius of 3 degrees, which corresponds to about $60 \%$ and about $90 \%$ of the LAT Point Spread Function at energy greater than $5 \mathrm{GeV}$ for the P7TRANSIENT and P7SOURCE class [14], respectively. More on this aspect is in Section 3.3.

The times and energies of detection of GRB130427A photons with energy in excess of $5 \mathrm{GeV}$ are shown in Figure 2.

2.2. A Scenario for Quantum-Spacetime. A rich phenomenological program was developed over the last decade on the basis of results for models of spacetime quantization suggesting that (see, e.g., [15-19]) it is possible for the quantum properties of spacetime to introduce small violations of the special relativistic properties of classical spacetime. A particularly valuable opportunity for phenomenology is provided by pictures of quantum-spacetime in which the time needed for a ultrarelativistic particle (of course the only regime of particle propagation that is relevant for this paper is the ultrarelativistic regime, since photons have no mass and the neutrinos we will consider in Section 4.5 have energies such that their mass is negligible) to travel from a given source to a given detector is $t=t_{0}+t_{\mathrm{QG}}$. Here $t_{0}$ is the time that would be predicted in classical spacetime, while $t_{Q G}$ is the contribution to the travel time due to quantum properties of spacetime. For energies much smaller than $M_{\mathrm{QG}}$, the characteristic scale of these quantum-spacetime effects, one expects that at lowest order $t_{\mathrm{QG}}$ is given by [20]

$$
t_{\mathrm{QG}}=-s_{ \pm} \frac{E}{M_{\mathrm{QG}}} \frac{D(z)}{c}
$$



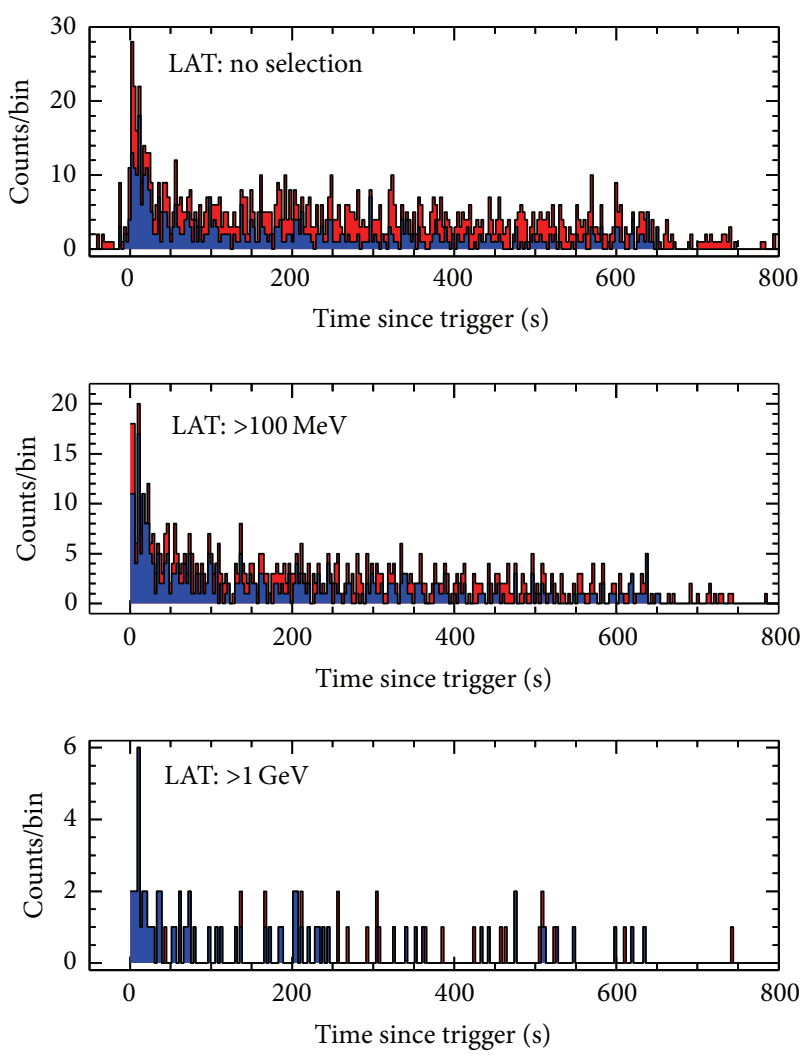

FIGURE 1: Fermi-LAT light curves for GRB130427A (bin size of 3 seconds). Photons selected within 10 degrees of the GRB130427A optical position. The contribution from the subset of photons within 3 degrees of the GRB130427A optical position is shown in blue.

where

$$
D(z)=\frac{c}{H_{0}} \int_{0}^{z} d \zeta \frac{(1+\zeta)}{\sqrt{\Omega_{\Lambda}+(1+\zeta)^{3} \Omega_{m}}} .
$$

Here the information cosmology gives us on spacetime curvature is coded in the denominator for the integrand in $D(z)$, with $z$ being the redshift and $\Omega_{\Lambda}, H_{0}$, and $\Omega_{m}$ denoting, as usual, respectively, the cosmological constant, the Hubble parameter, and the matter fraction. The "sign parameter" $s_{ \pm}$, with allowed values of 1 or -1 , as well as the scale $M_{\mathrm{QG}}$, would have to be determined experimentally. We must stress however that most theorists favor naturalness arguments suggesting that $M_{\mathrm{QG}}$ should take a value that is rather close to the "Planck scale" $M_{\text {planck }}=\sqrt{\hbar c^{5} / G_{N}} \simeq 1.22 \cdot 10^{19} \mathrm{GeV}$.

The picture of quantum-spacetime effects summarized in (1) does not apply to all quantum-spacetime models. One can envisage quantum-spacetime pictures that do not violate Lorentz symmetry at all, and even among the most studied quantum-spacetime pictures that do violate Lorentz symmetry one also finds variants producing (see, e.g., $[15,21,22]$ ) features analogous to (1) but with the ratio $E / M_{\mathrm{QG}}$ replaced by its square, $\left(E / M_{Q G}\right)^{2}$, in which case the effects would be much weaker and practically undetectable at present.

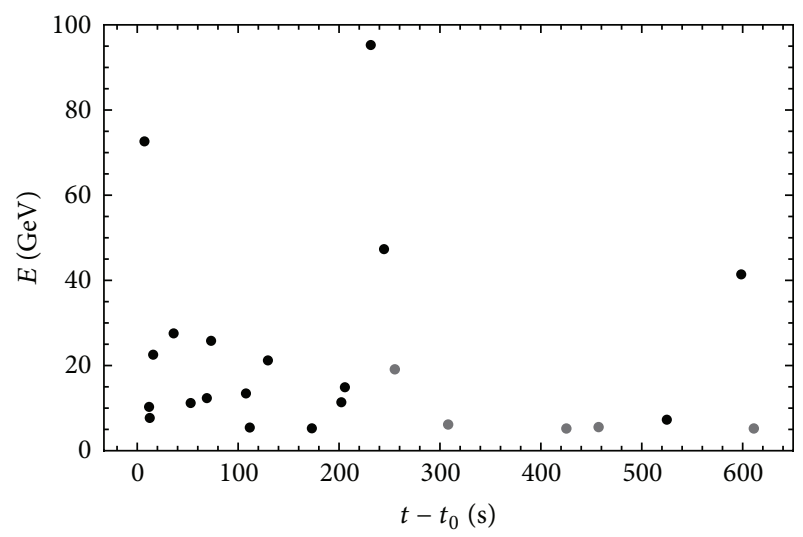

FIGURE 2: Times and energies of detection of GRB130427A photons with energy in excess of $5 \mathrm{GeV}$, selected within 10 degrees of the GRB130427A optical position. Darker points are for photons observed from within 3 degrees of the GRB130427A optical position.

It is important that some quantum-spacetime models allow for laws roughly of the type (1) to apply differently to different types of particles. For example, in the so-called "Moyal noncommutative spacetime," which is one of the most studied quantum-spacetimes, it is remarkably found [23] that the implications of spacetime quantization for particle propagation end up depending on the standardmodel charges carried by the particle and its associated coupling to other particles.

But let us stress, since it will be relevant later on in our discussion, that, while particle-dependent effects would indeed not be surprising, we know of no model that predicts an abrupt onset of such effects: the quantum-spacetime model building so far available offers candidates for particledependent effects, but in none of the known models the effects are exactly absent at low energy and then switch on to full strength above some threshold value of energy. Formula (1) illustrates the type of mechanisms that are known in the quantum-spacetime literature, with the effects confined to the ultraviolet by the behaviour of analytic functions introducing energy dependence such that the effects are small at low energies and become gradually stronger at higher energies.

This point is important since there is an apparently robust limit at $M_{\mathrm{QG}}>1.2 M_{\text {Planck }}$ derived in [24] (also see [22, 2528]) applying two different techniques to GRB090510 data. One technique, in which photons with energy below $5 \mathrm{GeV}$ play the primarily role, is based primarily on statistical properties of the arrival-time versus energy of photons, whereas the other technique exploits mainly the good coincidence of detection times between a single $31 \mathrm{GeV}$ photon and a very short duration burst (miniburst) of GRB090510 at lower energies. It should also be noticed that at energies higher than the range of energies we shall consider for GRB130427A the implications of (1) have been studied through observations of blazars by ground telescopes such as MAGIC and HESS, and this also produces $[27,29,30]$ significant bounds on $M_{\mathrm{QG}}$ at a level just below the Planck scale. In light of this the initial objective of our study was to establish whether the unusual 
quality of GRB130427A data could be used to study the effects of (1) with sensitivity providing constraints on the energy scale even slightly above the Planck Mass.

\section{Soft Spectral Lags for Minibursts within GRB130427A}

3.1. Spectral Lags for Minibursts within GRB130427A. As evident from what we already summarized in Section 2.2, part of our interest in GRB130427A originates from it being an opportunity for looking, at higher energies than in previous GRBs, for spectral lags of the type predicted for GRBs on the basis of (1). Let us however postpone commenting on the possible quantum-spacetime picture and (1) and instead contemplate first from a more general perspective the possibility of spectral lags with linear dependence on energy. Looking from this perspective at the GRB130427A data summarized in Figure 1, including only photons with energy greater than $5 \mathrm{GeV}$, one cannot fail to notice a very clear hint of systematic spectral lags. This is highlighted in Figure 3: for each of the four highest-energy photons in GRB130427A we find at least one more photon which lies on the same "spectral lag line," for a fixed choice of slope of such lines. The central line of Figure 3 is impressive because it suggests that 3 of the top- 9 most energetic photons in GRB130427A lie on one of such parallel spectral-lag lines.

Another way to visualize the content of Figure 3 is to consider the 7 points on those 3 lines and fit the values of the time translations needed to superpose the 3 lines. The result of this fit is shown in Figure 4, where we also show the single line which then best fits the 7 points.

For the slope one finds $-2.81 \pm 0.27 \mathrm{GeV} / \mathrm{s}$. We observe that from the perspective of (1) this slope of about $-3 \mathrm{GeV} / \mathrm{s}$ could be described as the case with $s_{ \pm}=1$ and $M_{\mathrm{QG}} \simeq 4$. $10^{-2} M_{\text {planck }}$.

3.2. Supporting Evidence from a Correlation Study. The observation visualized in Figures 3 and 4 is evidently noteworthy but it is not easy to quantify from it a statistical significance of the feature we are contemplating. Therefore we have performed a correlation study. We essentially studied the frequency of occurrence of linear spectral lags in our sample, that is, the number of times a line passing from a high-energy photon intercepts (within errors) a lower energy photon (in any case with energy greater than 5 or $15 \mathrm{GeV}$ ). This ultimately gives us an alternative way for determining the slope of lines such as those in Figure 3 that fit well the spectral lags present in the data, allowing for an easy estimate of statistical significance.

Our result for this correlation study is summarized in Figure 5, which gives a result in perfect agreement with the message conveyed in Figures 3 and 4 of the previous subsection, including the determination of the slope $d E / t$ of about -3 in $\mathrm{GeV}$ per second. Light dashed face, bold dashed, and dotted lines in Figure 5 represent, respectively, the $10 \%, 1 \%$, and $0.23 \%$ probability that the given frequency of occurrence of spectral lags is a chance occurrence. The lines have been calculated using 10000 Monte Carlo simulations

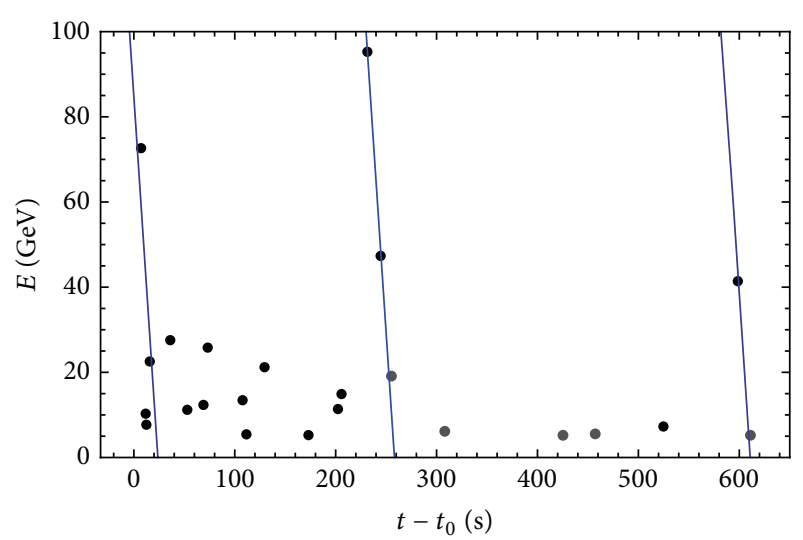

FIGURE 3: The three parallel straight lines here shown identify cases where photons on one such worldline could have been even emitted simultaneously (up to experimental errors) if (1) holds at the relevant energies.

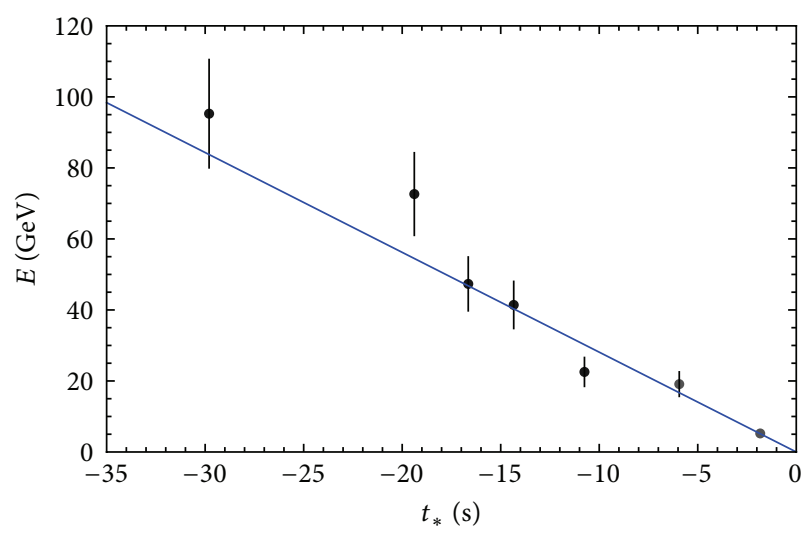

FIGURE 4: Here we show the results of a fit that superposes the three parallel lines in Figure 3. The seven points here shown are the ones on those parallel lines of Figure 3.

of photon energy versus time realizations assuming the same photon energy distribution as the data. We run the simulations by either randomizing the time arrival of the highenergy photons or scrambling them, obtaining similar results (Figure 5 shows the case with randomization of arrival times). Figure 5 shows that the probability of chance occurrence of spectral lags of about $3 \mathrm{GeV} /$ second is $\lesssim 0.23 \%$ for an energy threshold of $15 \mathrm{GeV}$ and just below the $1 \%$ level for an energy threshold of $5 \mathrm{GeV}$.

This comparison between our correlation study with a $5 \mathrm{GeV}$ lower cutoff and our correlation study with a $15 \mathrm{GeV}$ lower cutoff also suggests that the feature we are exposing emerges at high energies. This after all is also the message of Figure 3 where one immediately sees a pattern of spectral-lag correlations among the highest-energy photons and between the highest energy photons and some lower-energy photons, whereas such correlations among pairs of photons both with energy smaller than $15 \mathrm{GeV}$ are not clearly visible (if at all present). We confirm this message with the study visualized in Figure 6 which is of the same type reported in Figure 5 but 


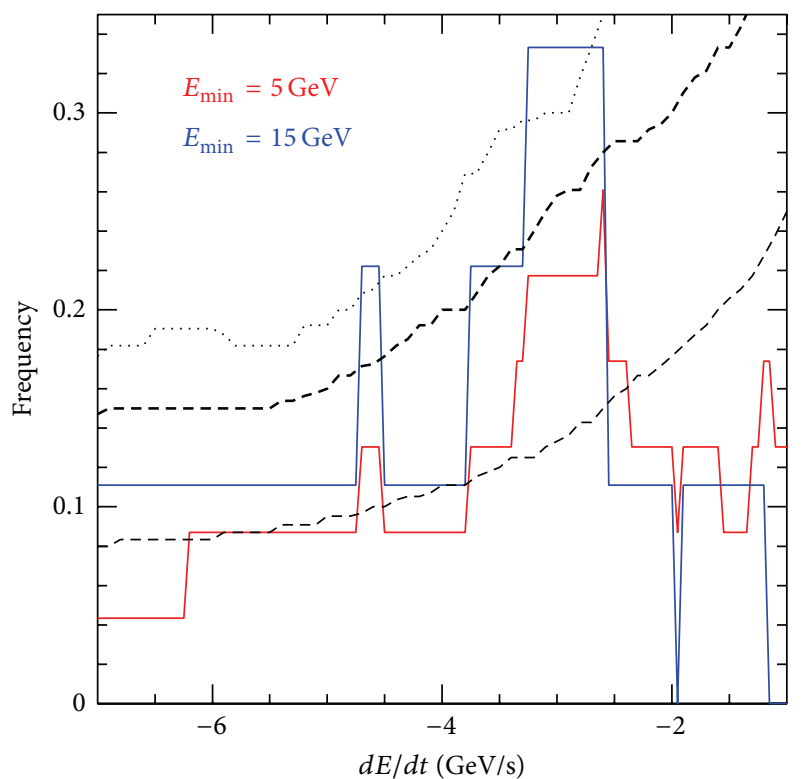

FIGURE 5: Here shown is a study of the frequency of occurrence of spectral lags in our sample as a function of a minimum photon energy and without any prior assumption on high energy photons. The red (resp., blue) solid line describes the frequency of occurrence of a certain spectral-lag slope within the sample of GRB130427A events with energy grater than $5 \mathrm{GeV}$ (resp., $15 \mathrm{GeV}$ ). Dotted/dashed lines represent the $10 \%, 1 \%$, and $0.23 \%$ probability that the given frequency of occurrence of spectral lags is a chance occurrence. The peak of the $d E / d t$ distribution is consistent with the estimate of $-2.8 \mathrm{GeV} / \mathrm{s}$ obtained from the best-fit analysis described in Figure 4.

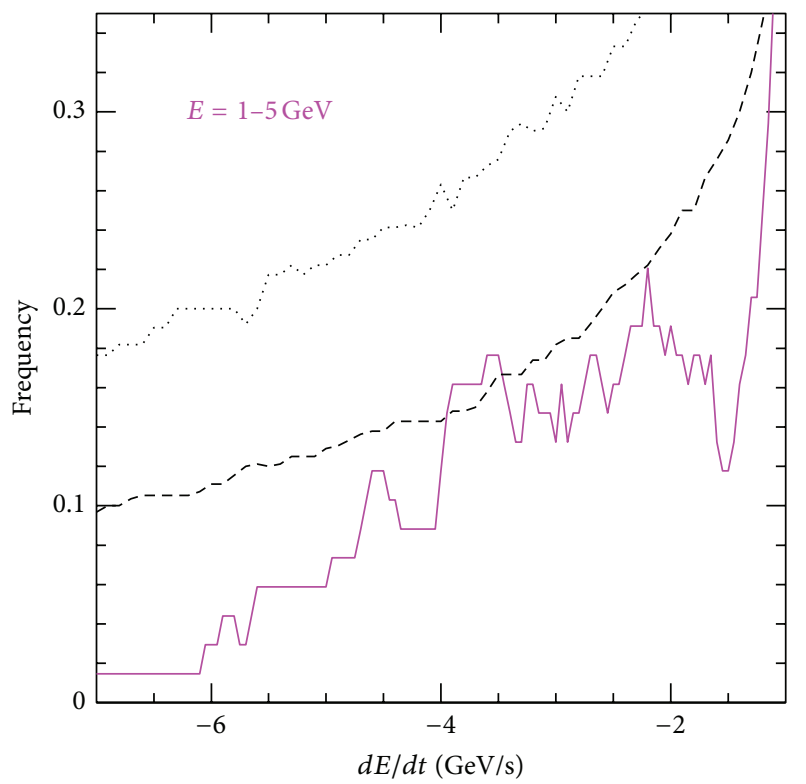

FIGURE 6: Here shown are the results of a study of the type already described in Figure 5, but now restricting the analysis to photons with energy between $1 \mathrm{GeV}$ and $5 \mathrm{GeV}$. Again the dotted line and the dashed line estimate, respectively, the $1 \%$ and $10 \%$ probability that the given frequency of occurrence of spectral lags is a chance occurrence. focuses on GRB130427A photons with energy between 1 and $5 \mathrm{GeV}$ : contrary to what was shown in Figure 5 we find no significant signal in the study shown in Figure 6.

3.3. Aside on the Structure at Late Times. It might be noteworthy that the statistical significance of our analysis increases if we focus on late times in the development of GRB130427A. To see that this is the case we look at the data points in Figure 3 focusing on a 450-second interval, from 190 seconds to 640 seconds after the trigger. What we find is shown in Figure 7. Comparing Figure 7 to Figure 5 one sees clearly that after the first 190 seconds the evidence of spectral lags with a slope of about $-3 \mathrm{GeV} / \mathrm{s}$ is significantly stronger. In particular, when focusing on times after the first 190 seconds also the study of correlations taking into account all photons with energy greater than $5 \mathrm{GeV}$ produces an outcome whose probability of chance occurrence is significantly less than $0.23 \%$.

Considering the potential significance of the findings reported in Figure 7 it is appropriate here to pause for an assessment of the possibility that background events might be affecting the rather striking evidence we are gathering. In this respect it is noteworthy that two events among those that contribute to the result of Figure 7 were observed at more than 3 degrees (but less than 10 degrees; see Section 1) off the optical position of GRB130427A. We must therefore attribute to them a somewhat higher risk (higher than for those observed within 3 degrees of GRB130427A) of being background events. These two events are the $19 \mathrm{GeV}$ event visible in the bottom central part of Figure 3 at about 250 seconds after trigger and the $5.2 \mathrm{GeV}$ event visible in the bottom right part of Figure 3 at about 610 seconds after trigger.

In Figures 8 and 9 we offer a quantification of how different the outlook of our analysis would be when considering the possibility of excluding from it one of these two events. Comparing Figure 8 to Figure 7 one sees that renouncing to the $5.2 \mathrm{GeV}$ event has tangible but not very significant implications for our analysis. Comparing Figure 9 to Figure 7 one sees that renouncing to the $19 \mathrm{GeV}$ event does have rather significant implications for our analysis, but our findings would be noteworthy even without the $19 \mathrm{GeV}$ event.

In light of this special role played by the $19 \mathrm{GeV}$ event, we have estimated the probability of a background event of energy between 15 and $30 \mathrm{GeV}$ within the 450 -second window considered for Figure 7 . We find that this probability is of about $15 \%$ by both modeling the background and using data available in a temporal and directional neighborhood of GRB130427A. It appears legitimate to also notice that if such a $19 \mathrm{GeV}$ event was truly background and therefore unrelated to GRB130427A it could have come at any time within that 450second window, but it turned out to contribute so strongly to the significance of our analysis only because it happened to be an excellent match for the central straight line in Figure 3. In order for it to be such a strong contributor to the significance of our analysis it had to be detected within a temporal window around that straight line of about 5 seconds, so we are dealing with a $15 \%$ probability overall but only $a \sim 0.17 \%$ probability of a background event contributing that strongly to our case. 


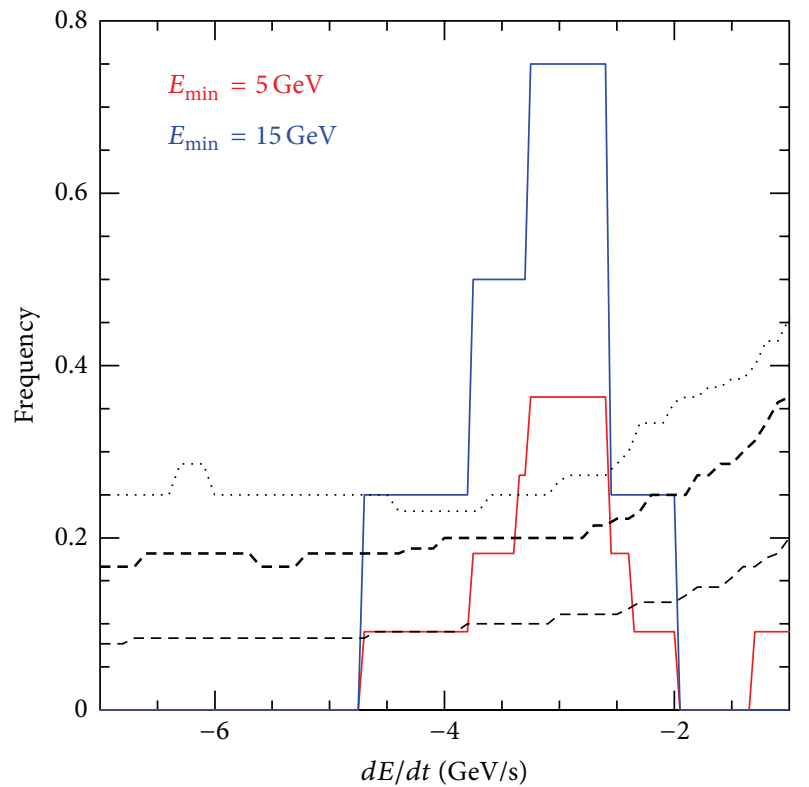

FIGURE 7: Here shown are the results of a study of the type already described in Figure 5, but now restricting the analysis to the time interval from 190 seconds to 640 seconds after the trigger. Dotted/dashed lines represent again the $10 \%, 1 \%$, and $0.23 \%$ probability that the given frequency of occurrence of spectral lags is a chance occurrence.

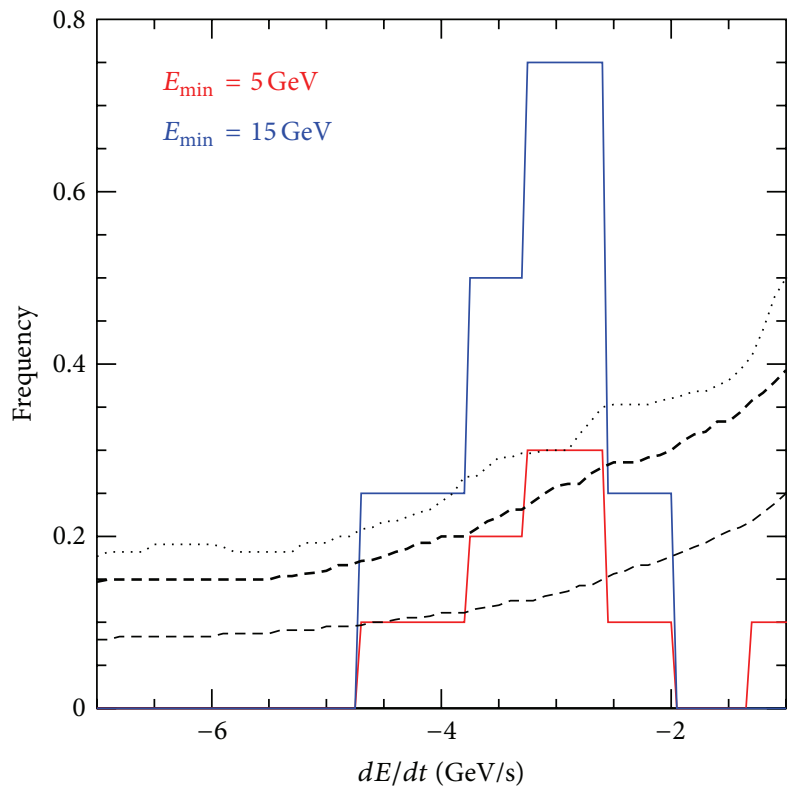

FIGURE 8: Here shown are the results of a study exactly of the type already described in Figure 7 (including the restriction to the time interval from 190 seconds to 640 seconds after the trigger), but now excluding from the analysis a $5.2 \mathrm{GeV}$ event from about 610 seconds after trigger. Dotted/dashed lines represent again the $10 \%, 1 \%$, and $0.23 \%$ probability that the given frequency of occurrence of spectral lags is a chance occurrence.

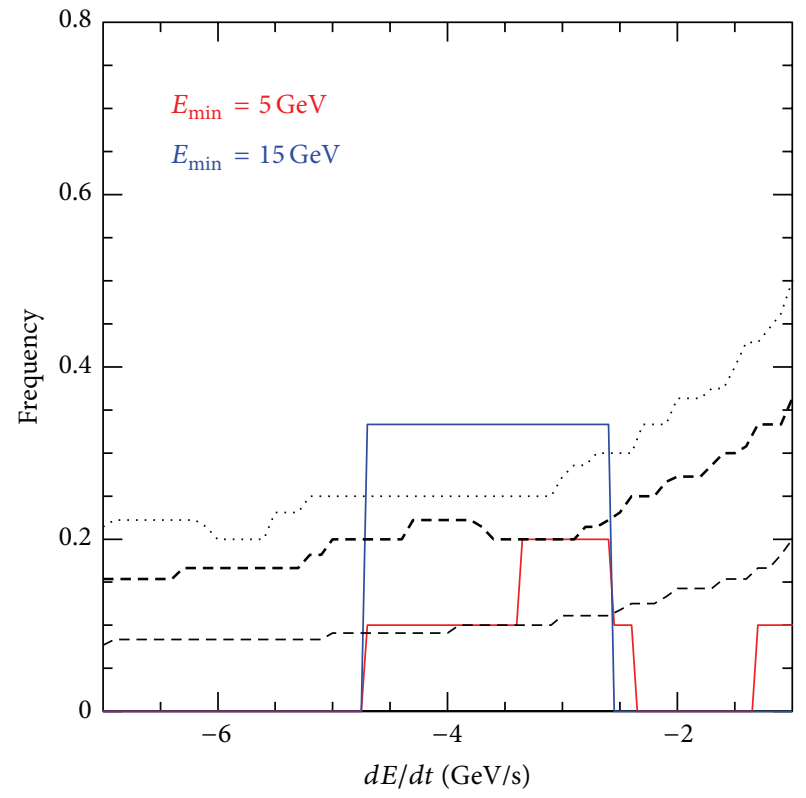

Figure 9: Here shown are the results of a study exactly of the type already described in the previous Figure 7 (including the restriction to the time interval from 190 seconds to 640 seconds after the trigger), but now excluding from the analysis a $19 \mathrm{GeV}$ event from about 250 seconds after trigger. Dotted/dashed lines represent again the $10 \%, 1 \%$ and $0.23 \%$ probability that the given frequency of occurrence of spectral lags is a chance occurrence.

(Note however that we are assuming "a posteriori" that the $19 \mathrm{GeV}$ photon is correlated with the 95 and $50 \mathrm{GeV}$ events.)

As stressed above, if we were to exclude the $19 \mathrm{GeV}$ event we would still be left with the noteworthy outcome shown in Figure 9. And evidently the blue line in Figure 9 is independent of the contribution by the $5.2 \mathrm{GeV}$ event. It would require a significant "conspiracy" for it to be the case that both the $19 \mathrm{GeV}$ event and the 5.2 event were background events which happened to be detected just at the right times to improve the outlook of our analysis.

\section{Seeking Further Evidence and Redshift Dependence}

We are assuming that looking at GRB130427A is like looking at GRBs in high definition. This allowed us to notice some unexpected features in the previous section, and we shall now try to use this guidance to go back to previous bright high-energy GRBs and try to recognize the same features. As we do this we shall also monitor any indication of redshift dependence of these features. It would be of particular interest from a quantum-spacetime perspective if we could find encouragement for redshift dependence governed by the $D(z)$ of (1), but we shall remain open to other options (and we shall end up favouring the hypothesis of description of the features presented in this work not based on quantumspacetime pictures). 
4.1. The Case of GRB080916C. GRB080916C is our first attempt to recognize in another GRB the features here uncovered for GRB130427A. This is a very interesting GRB for our purposes, particularly for the issue of possible redshift dependence, since GRB080916C was $[28,31]$ at redshift of 4.35, much higher than the 0.34 of GRB130427A. And GRB080916C must be viewed as a very powerful high-energy GRB, if one indeed reassesses its LAT fluence in light of its high redshift.

There is at least one more criterion which would characterize GRB080916C as a GRB in the Fermi-LAT catalogue whose comparison to GRB130427A is particularly significant from the viewpoint of the analysis we are here reporting: GRB080916C contains a $13.3 \mathrm{GeV}$ photon, a $7 \mathrm{GeV}$ photon, and a $6.2 \mathrm{GeV}$ photon, and when converting these energies at time of detection into energies at time of emission, taking into account the large redshift of 4.35 , one concludes that all these 3 photons had energy at time of emission of more than $30 \mathrm{GeV}$. This compares well with one aspect which we stressed about GRB130427A, which has 4 photons above $30 \mathrm{GeV}$ (actually above $40 \mathrm{GeV}$ ).

It is clear that the presence, in a GRB, of photons of such high inferred energies at emission time does not mean that we are assured to see one of our "hard minibursts with spectral lags." On the contrary the probability of such success remains rather low, since surely often when we see 3 or 4 such photons they belong to different hard minibursts within the GRB. To see how this is the case imagine that from GRB130427A we only observed 3 rather than 9 photons with energy greater than $19 \mathrm{GeV}$ : if we take off randomly 6 photons among the top-9 most energetic ones from Figure 3 no hard miniburst would be noticeable. But in some cases we are bound to get lucky, and this is what at least appears to be the case with GRB080916C, as shown in Figure 10, which reports the events for GRB080916C with energy higher than $1 \mathrm{GeV}$.

Our readers should easily notice from Figure 10 that some features we exposed earlier in this paper for GRB130427A are present also in this GRB080916C case. In particular, the highest energy photon $(13.3 \mathrm{GeV})$ is detected as first photon after the longest time interval of "high-energy silence" during the first 25 seconds. And then some other highenergy photons are detected soon after this highest-energy photon. So actually GRB080916C provides us with a very clear example of the feature we are proposing, describable as early hard development of minibursts within a long burst.

Next we should use GRB080916C as an opportunity for finding further evidence of linear spectral lags. A challenge for this of course comes from the fact that the slope of these spectral lags could depend on redshift. In the quantumspacetime picture of (1) the redshift dependence would be governed by the $D(z)$ of (1), but if (as we shall end up favouring) the linear spectral lags hve astrophysical origin then various forms of redshift dependence could be conjectured (a promising hypothesis will be discussed in Section 5, but several alternatives could be considered).

In order to test the hypothesis of redshift dependence governed by the $D(z)$ of $(1)$ one should do as in Figure 10, that is, rescale the slope taking into account the difference of redshifts. According to (1) the relationship between

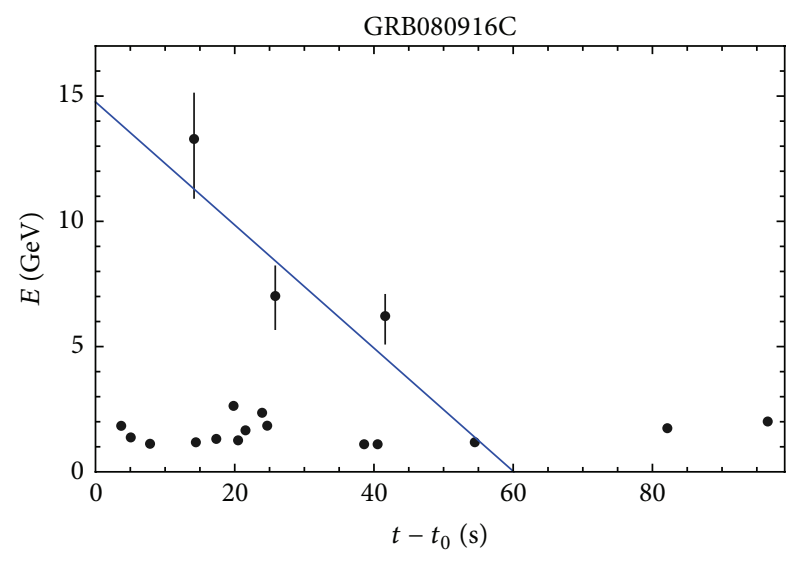

FIgure 10: Here shown is the Fermi-LAT sequence of photons with energy greater than $1 \mathrm{GeV}$ for GRB080916C. We visualize error bars at the $15 \%$ level for the energies of the most energetic events, which play a special role from our perspective. The slope of the straight line here shown is not fit on this data but rather is obtained from the slope fit in Figure 4 (for GRB130427A) by rigidly rescaling it by the factor of $D(0.34) / D(4.35)$ dictated by (1).

the GRB080916C slope and the GRB130427A slope is fixed to be $D(0.34) / D(4.35)$. The slope of straight lines characteristic of high-energy spectral lags should be then for GRB080916C much lower than for GRB130427A. A straight line with such a slope is shown in Figure 10 and it gives a rather intriguing result: that straight line obtained from the ones of Section 3 by correcting for the redshift, assuming the validity of (1), is consistent with as many as 3 other GRB080916C events. And one should notice that among the 4 events that would reasonably fit this straight line one finds the 3 highestenergy photons which we highlighted at the beginning of this subsection.

As mentioned, if the features we are uncovering do not have quantum-spacetime origin (and therefore are pureastrophysics effects) then one would not expect redshift dependence governed by the $D(z)$ of (1). Rather than speculating here about the possible forms of redshift dependence that astrophysics could produce, we choose to explore the issue by simply assuming redshift (and context) independence of the slope of spectral lags within hard minibursts. This simple hypothesis is probably unrealistic, but at least it gives us a hypothesis alternative to the redshift dependence of (1) and this will help us to assess the significance of what we find assuming the redshift dependence of (1). In Figure 11 we let go through the point (with error bar) corresponding to the highest-energy photon a straight line with the slope we determined in the previous section.

Figure 11 shows that by not rescaling the slope for redshift one establishes no connection between the highestenergy photon and the second and third most energetic photons in the sample. This is disappointing for the redshiftindependence hypothesis. But of course it may well be that the remarkable feature highlighted in Figure 10, for (1)governed-redshift case, is merely accidental and instead a true 


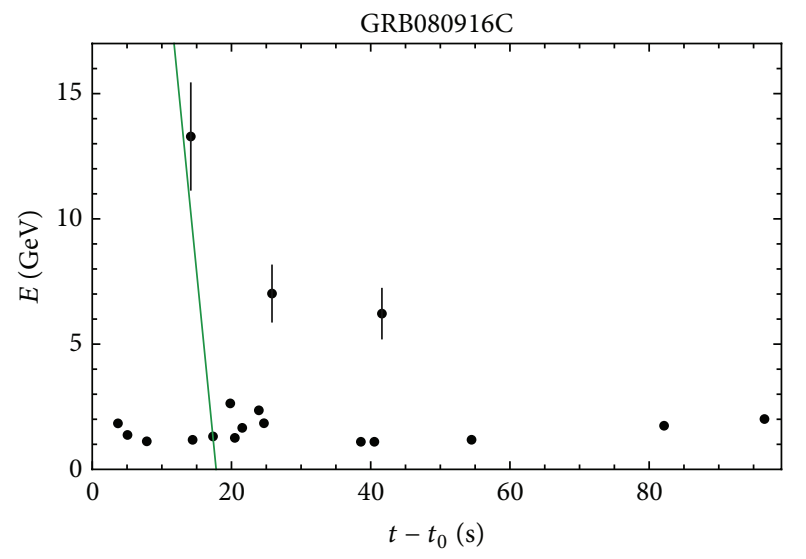

FIGURE 11: Here shown are the same data points already shown in Figure 10, but now the straight line added on top of the data point has exactly the slope we derived in Section 3 (Figure 4), without any rescaling.

connection is the one visible in Figure 11 for the redshiftindependence hypothesis, with a linear soft spectral lag between the highest-energy photon and the photon detected immediately after it.

Still it is noteworthy that if one relies on just the comparison of Figures 10 and 11 the conclusion must be that GRB080916C scores a point for the redshift dependence governed by the $D(z)$ of $(1)$.

4.2. The Case of GRB090926A. GRB090926A is another long burst of the pre-GRB130427A Fermi-LAT catalogue that compares well in terms of the brightness of the high-energy component with GRB080916C. And GRB090926A was at a redshift $[28,31]$ of 2.106 , so that it provides a case with a value of redshift somewhere in between the ones for GRB130427A and GRB080916C.

In considering GRB090926A we restrict our focus on the photons shown in Figure 12, with energy greater than $1.5 \mathrm{GeV}$. Again in Figure 12 we see that for GRB090926A (just as observed above for GRB130427A and GRB080916C) there is a clearly noticeable "hard miniburst" with early hard development whose onset coincides with the highest-energy photon in the whole signal: the figure shows several photons rather densely distributed between 6 and 21 seconds after trigger; then "hard-photon silence" for 5 seconds, and at 26 seconds after trigger the highest-energy photon in the burst is observed, followed by other high-energy photons.

Concerning the other feature of our interest, involving the presence of soft spectral lags which are linear in energy and the possible redshift dependence of this linear law, in the spirit of what we did in the preceding subsection we show in Figure 12 straight lines on which the highest-energy photon lies (within errors). In Figure 12(b) the line has exactly the same slope derived in Section 3 (our redshift-independent scenario) and it is notable that the first photon in our sample after the highest-energy photon lies on that line. But it is

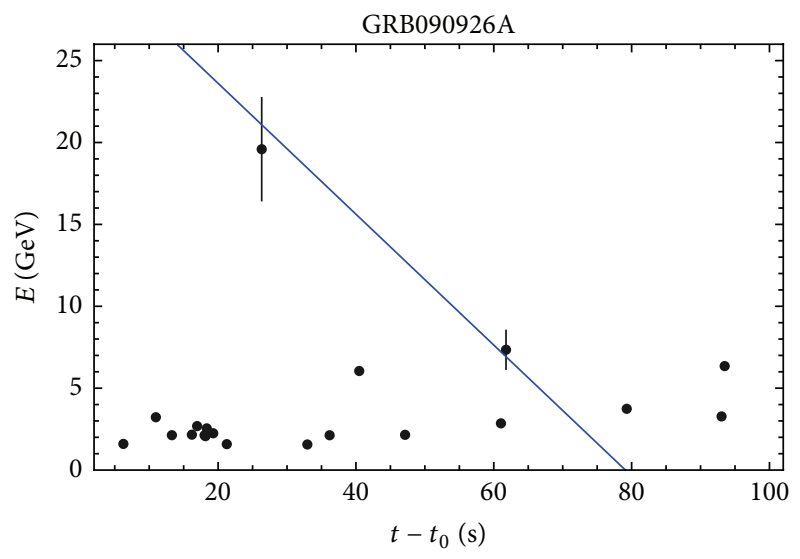

(a)

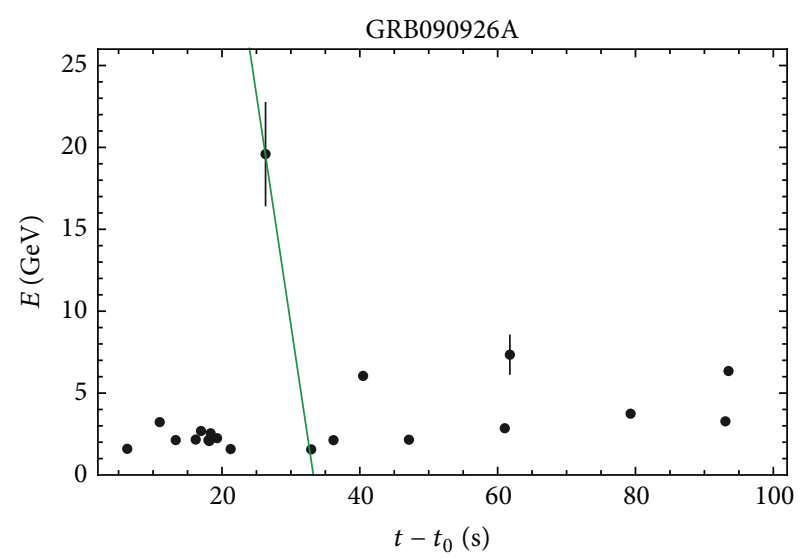

(b)

FIGURE 12: Here shown is the Fermi-LAT sequence of photons with energy greater than $1.5 \mathrm{GeV}$ for GRB090926A. We visualize an error bar at the $15 \%$ level for the energy of the two most energetic events. (b) A straight line with the slope we derived in Section 3. (a) A straight line with slope obtained from the slope derived in Section 3 by rigidly rescaling it by the factor of $D(0.34) / D(2.106)$ (2.106 being the redshift of GRB090926A) dictated by our (1).

Figure 12(a) which apparently contains the most intriguing message: both in GRB130427A and in GRB080916C the slope predicted by (1) for the same value of $M_{\mathrm{QG}}$ (and taking into account the redshift) gave a straight line compatible with the highest-energy photon and two other of the most energetic photons in the bursts, and here again, as shown indeed in Figure 12, the same strategy of analysis places on the same spectral-lag straight line the highest-energy photon and the second-highest-energy photon of GRB090926A. Again the slope of the straight line shown in Figure 12(a) was obtained by rigidly rescaling the GRB130427A slope found in Figure 4 by the factor $D(0.34) / D(2.106)$ as dictated by the redshift dependence of (1).

4.3. The Case of GRB090902B. GRB090902B, which was at a redshift $[28,31]$ of 1.822 , is the other long burst in the pre-GRB130427A Fermi-LAT catalogue that compares well in 
terms of the brightness of the high-energy component with GRB080916 and GRB090926A.

As stressed already in the previous subsection, even in presence of a few high-energy photons one has no guarantee to see in the data a single hard miniburst containing more than one of the highest-energy photons. More often than not the few highest-energy photons Fermi does catch will have originated from different hard minibursts within the burst. In light of this it may appear that we were unreasonably lucky in Figure 10 for GRB080916C and in Figure 12(a) for GRB090926A. This unreasonably high level of success of our ansatz does not apply to the case of GRB090902B which we are now considering: as we show in Figure 13(a), focusing on GRB090902B photons with energy greater than $1 \mathrm{GeV}$, our spectral-lag straight lines, with slope computed from the one derived in Section 3 according to the redshift dependence coded in (1) (the correction factor for the slope in this case being $D(0.34) / D(1.822)$ ), do not connect the highest-energy photon with any other one of the most energetic photons in GRB090902B. Figure 13(a) does show that the highestenergy photon of GRB090902B is reasonably compatible within our ansatz with a group of five photons with energy $1<E \lesssim 5 \mathrm{GeV}$ detected between 100 and 125 seconds after trigger, but this is far less impressive than what we found for GRB130427A, GRB080916C, and GRB090926A.

As shown in Figure 13(b), even in the case of GRB090902B the redshift-independence hypothesis does not provide a better match to the data than the hypothesis of redshift dependence governed by (1).

Particularly noteworthy is the fact that even in the case of GRB090902B we can observe again the structure of our "hard minibursts": the sequence of high-energy photons has a relatively long silent time interval between the highest-energy photon and the photon that precedes it (long compared to the typical time interval between subsequent detections of these GRB090902B high-energy photons). So the highest-energy photon is detected after a sizable time interval of high-energy silence and then a few high-energy photons are detected soon after.

4.4. The Case of a Short Burst: GRB090510. Our analysis of the four Fermi-LAT bursts with most significant highenergy signal, GRB130427A, GRB080916C, GRB090902B, and GRB090926A, gave rather consistent indications. At least one miniburst with early hard development was found in all of them in correspondence of the highest-energy photon in the GRB. And in all four cases the onset of this miniburst containing the highest-energy photon was preceded by a relatively large time interval of high-energy silence: one finds in all these four GRBs a suitable lower cutoff on the energies of the photons to be included in the analysis such that there are no detections of photons in such a sample for a certain time interval before the detection of the highest-energy photons, while going at still earlier times one finds a denser rate of detections of photons in the sample.

Concerning the quantification of the soft spectral lags within such minibursts in terms of a linear law of dependence on energy GRB080916C, GRB090902B, and GRB090926A

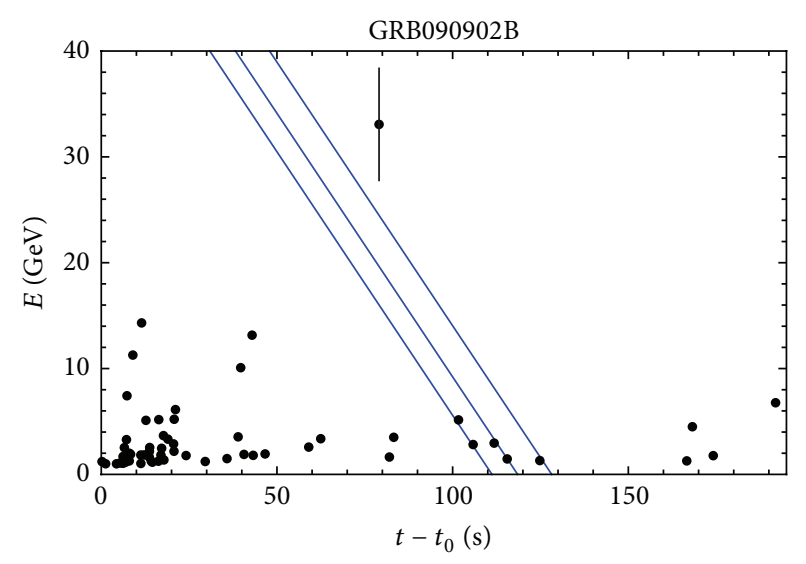

(a)

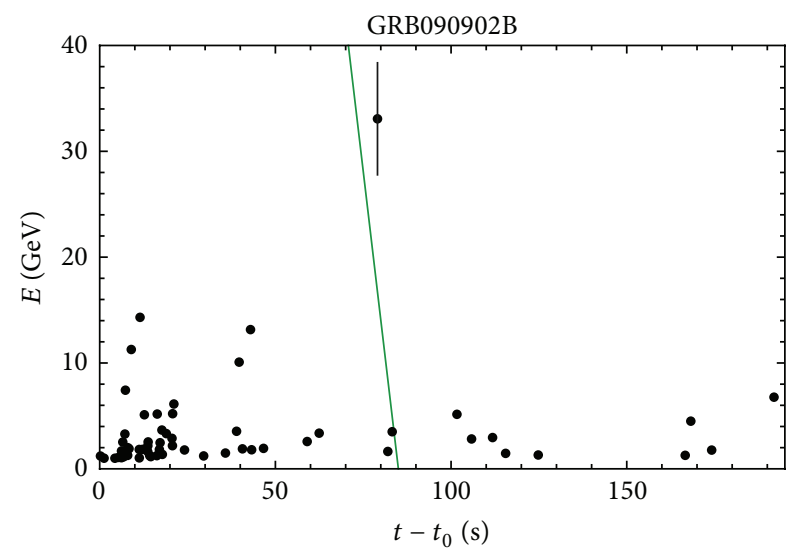

(b)

FIGURE 13: Here shown is the Fermi-LAT sequence of photons with energy greater than $1 \mathrm{GeV}$ for GRB090902B. We visualize an error bar at the $15 \%$ level for the energy of the most energetic event. (b) A straight line with the slope we derived in Section 3. (a) Some straight lines with slope obtained from the slope derived in Section 3 by rigidly rescaling it by the factor of $D(0.34) / D(1.822)$ dictated by our (1) for a GRB at redshift of 1.822.

did not add any particularly decisive evidence but offered an overall consistent picture. As stressed above (particularly for GRB080916C and GRB090926A) this additional supporting evidence for the linear quantification of the soft spectral lags was consistent with the possibility of redshift dependence governed by (1), as required by the quantum-spacetime hypothesis. However, the next burst we now want to analyze will lead us to favour overall the interpretation as intrinsic properties of the long GRBs. This burst that we want to analyze next is the only short bursts with strong high-energy signal in the Fermi catalogue5: GRB090510 whose sequence of detection times for photons with energy greater than $1 \mathrm{GeV}$ is shown in Figure 14.

In this case let us start by discussing our test with the spectral-lag straight lines derived in Section 3 from GRB130427A data. As shown in Figure 14 one does find some potential candidates as high-energy photons lagging the highest energy photon according to the quantification we 
first derived in Section 3, but with respect to the outcome of the analogous tests we did previously on long bursts in this case the outlook is less promising: both for the redshift-independent hypothesis and for (1) based redshiftdependent hypothesis the test does not fail badly but it also gives only marginally plausible candidates. This is because in Figure 14 one notices that the highest-energy photon lies in time-of-detection quasi-coincidence with several other high-energy photons. It is hard to believe that this time coincidence is accidental (not a manifestation of an interrelation between the highest-energy photons an those other nearly coincident high-energy photons). Figures 14(a) and 14(b) would suggest that the highest-energy photon happens to have been detected in reasonably good time coincidence with those other high-energy photons but actually it is part of a miniburst involving photons detected at much later times. This is certainly possible, but we feel it is somewhat hard to believe.

We observe that in the Fermi-LAT data on GRB090510 there is only one photon with energy greater than $30 \mathrm{GeV}$ (indeed the 31-GeV event we discussed), and, considering that the redshift of GRB090510 was [28, 31] 0.897, there was no other photon in GRB090510 data set with energy greater than $30 \mathrm{GeV}$ even at time of emission.

4.5. An Observation about GRB Neutrinos. Having considered the brightest pre-GRB130427A Fermi-LAT GRBs we shall now offer also an observation about GRB neutrinos. This involves data of more uncertain status, but it highlights another striking quantitative match for one of the features we noticed in GRB130427A data. Actually this will also render manifest why we were in good position to notice such features in GRB130427A data, since this observation about GRB neutrinos was already reported by some of us in [32], before GRB130427A (the arXiv preprint version of [32] is from March 2013).

Reference [32] also contemplates the type of energy dependence of times of arrival that is produced by (1), exactly as we are doing in the present study. But [32] was aimed at the context of GRB-neutrino searches by IceCube.

Recently IceCube reported [33] no detection of any GRBassociated neutrino in a data set taken from April 2008 to May 2010. Reference [32] focuses however on 3 Icecube neutrinos that are described in publicly available studies $[33,34]$ and are plausible (though weak) GRB-neutrino candidates. These are [34] a $1.3 \mathrm{TeV}$ neutrino $1.95^{\circ}$ off GRB090417B, with localization uncertainty of $1.61^{\circ}$, and detection time 2249 seconds before the trigger of GRB090417B, a $3.3 \mathrm{TeV}$ neutrino $6.11^{\circ}$ off GRB090219, with a localization uncertainty of $6.12^{\circ}$, and detection time 3594 seconds before the GRB090219 trigger, and a $109 \mathrm{TeV}$ neutrino, within $0.2^{\circ}$ of GRB091230A, with a localization uncertainty of $0.2^{\circ}$, and detected some 14 hours before the GRB091230A trigger.

The fact that all 3 of these GRB-neutrino candidates were detected sizably in advance of the triggers of the GRBs they could be associated with is not particularly significant from the standard perspective of this sort of analysis, and actually obstructs any such attempt to view them as GRB

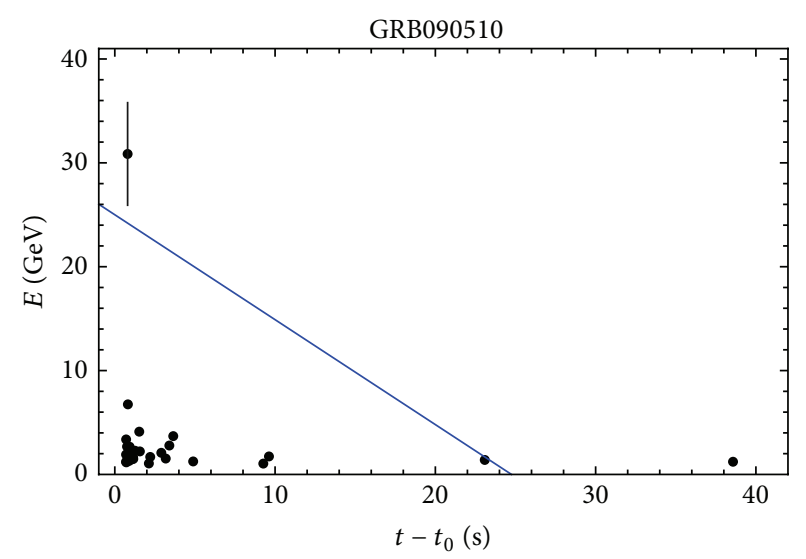

(a)

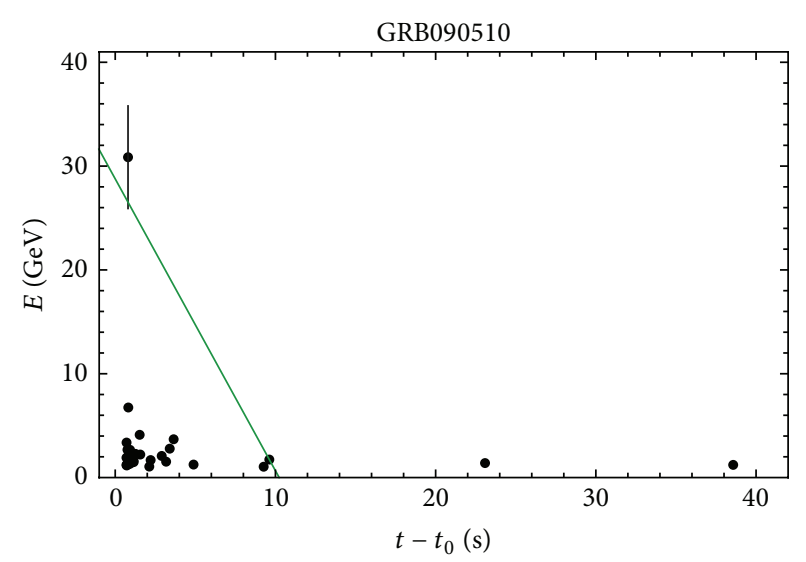

(b)

FIgURE 14: Here shown is the Fermi-LAT sequence of photons with energy greater than $1 \mathrm{GeV}$ for GRB090510. We visualize an error bar at the $15 \%$ level for the energy of the most energetic event. (b) A straight line with the slope we derived in Section 3. (a) A straight line with slope obtained from the slope derived in Section 3 by rigidly rescaling it by the factor of $D(0.34) / D(0.897)$ ( 0.897 being the redshift of GRB090510), as dictated by (1).

neutrinos: no current GRB model suggests that neutrinos could be emitted thousands of seconds before a GRB. But a collection of GRB-neutrino candidates all sizably in advance of corresponding GRB triggers is just what one would expect from (1) (for $s_{ \pm}=1$ ). Using this observation in [32] it was shown that possibly 2 of the 3 mentioned GRB-neutrino candidates could be actual GRB neutrinos, governed by (1).

The most appealing possibility discussed in [32] is for the 3.3 TeV and the $109 \mathrm{TeV}$ neutrinos to be actual GRB neutrinos and requires $M_{\mathrm{QG}} \simeq M_{\text {planck }} / 25$. The other possibility is for the $1.3 \mathrm{TeV}$ and the $3.3 \mathrm{TeV}$ neutrinos to be actual GRB neutrinos and requires an even smaller value of $M_{\mathrm{QG}}$, of about $M_{\mathrm{QG}} \simeq M_{\text {planck }} / 100$.

Evidently what we found in the present analysis inspired by and based in part on GRB130427A is at least to some extent consistent with what was found in [32] for the $3.3 \mathrm{TeV}$ and the $109 \mathrm{TeV}$ neutrinos. In order to be more precise in this assessment we must take into account the fact 
that the redshifts of GRB090219 and of GRB091230A were not determined. We shall deal with this in the next (closing) section by handling the uncertainty in the redshift as another source of uncertainty for testing (1) with these two neutrinos: since GRB090219 was a short burst we can reasonably [35] assume that its redshift was between 0.2 and 1 , whereas for GRB091230A, a long burst, we can reasonably [36] assume that its redshift was between 0.3 and 6 .

\section{Perspective on Results and Closing Remarks}

We here took as starting point the remarkable Fermi-LAT observation of GRB130427A. We looked at this GRB assuming that it was giving us a chance to see a long bright burst in high resolution. Using as guidance the quantum-spacetimeinspired ansatz of (1) we stumbled upon a few related features present in the highest-energy portion of the Fermi-LAT data GRB130427A. We then used this insight gained from the high-resolution GRB130427A to recover some evidence of the same features in the brightest previous Fermi-LAT observations of GRBs and even for some candidate GRB neutrinos.

Since we considered a few features in several contexts it is useful to close this paper by offering a perspective on our findings. We articulate this section into subsections, each one devoted to one of the features and aspects we have considered and ordered according to the strength of the supporting evidence which we found.

5.1. Structure of Hard Minibursts. The feature that emerged most robustly from our analysis concerns the presence of hard minibursts, whose onset is marked by the highestenergy photon observed in a given GRB. We found this feature (here illustrated in Figure 15) in all the top four brightest high-energy GRBs in the Fermi-LAT catalogue GRB130427A, GRB080916C, GRB090902B, and GRB090926A.

The minibursts become noticeable only when focusing the analysis on the few highest-energy photons in the signal: for GRB130427A it was clearly visible for photons with energy higher than $5 \mathrm{GeV}$, while for GRB080916C, GRB090902B, and GRB090926A it manifested itself when restricting the analysis to photons of energy higher than $1 \mathrm{GeV}$ or $2 \mathrm{GeV}$.

The structure of these hard minibursts was in all cases such that the high-energy signal (composed of photons selected with energy above a certain high value, as we just stressed) has a long silent time interval between the highestenergy photon and the high-energy photon that precedes it (long compared to the typical time interval between subsequent detections of these high-energy photons). So the highest-energy photon is detected after a sizable time interval of high-energy silence of the signal, and then a few highenergy photons are detected soon after.

Besides being identifiable in connection with the highest-energy photon of all the four brightest long FermiLAT GRBs, GRB130427A, GRB080916C, GRB090902B, and GRB090926A, in the case of GRB130427A one can tentatively identify two more of such hard minibursts, with exactly the same structure (see Figure 3).

We have some evidence for a specific behaviour of these soft spectral lags, with linear dependence on energy. This found rather sizable support in our analysis of GRB130427, as shown in Section 3.1 by exhibiting some rather compelling candidate photons for 3 hard minibursts and shown in Sections 3.2 and 3.3 by statistical analyses taking the shape of studies of the frequency of occurrence of a given type of spectral lags in our GRB130427A sample.

GRB130427A on its own provides a rather compelling case for the presence of these linear-in-energy soft spectral lags. And when we considered available data on other bright Fermi-LAT GRBs we did find some additional supporting evidence, though significantly weaker than in the GRB130427A case.

5.2. Strength and Weakness of the Quantum-SpacetimeInspired Hypothesis. When we looked to other bright FermiLAT GRBs as opportunities for additional evidence supporting our linear spectral lags it was necessary to contemplate a redshift dependence of the linear law. We had a candidate ready for the task, taking the shape of the redshift dependence codified by $D(z)$ for the linear spectral lags of (1). We explored the efficaciousness of this hypothesis by taking the linear energy-versus-time dependence derived on GRB130427A data and assuming that it should apply to other bursts upon rigidly rescaling the slope by $D(0.34) / D(z)$, (where $D(0.34)$ is for the redshift of 0.34 of GRB130427A).

A dramatic confirmation would have been to find for each burst several photons lining up (in energy versus time of detection) just according to the linear relationship, with slope rigidly rescaled by $D(0.34) / D(z)$. But this was clearly unlikely since, even for the bright long bursts, GRB080916C, GRB090902B, and GRB090926A the FermiLAT only detected a rather small overall number of photons with energy greater than a few $\mathrm{GeV}$. Nonetheless at least in two cases our investigation of this possibility appears to have been successful beyond expectations. For GRB080916C by rigidly rescaling the slope of our linear law by $D(0.34) / D(4.35)$ (with 4.35 for the redshift of GRB080916C) and insisting that the relevant straight line be consistent with the highest-energy photon, we found a rather noteworthy group of four photons with energy and detection times all consistent with our linear law for soft spectral lags. These are the four photons on the straight line in Figure 10, which include in particular the highest-energy photon and the second and third most energetic photons of GRB080916C. And in the analogous analysis of GRB090926A we found that our quantum-spacetime ansatz for the dependence on redshift of the linear spectral lags remarkably also placed in connection the two highest energy photons. While all this may well be accidental, the cases of GRB080916C and GRB090926A clearly can seen as providing encouragement for the law of redshift dependence of (1) and with it the possible quantum-spacetime interpretation of the features we here exposed. 


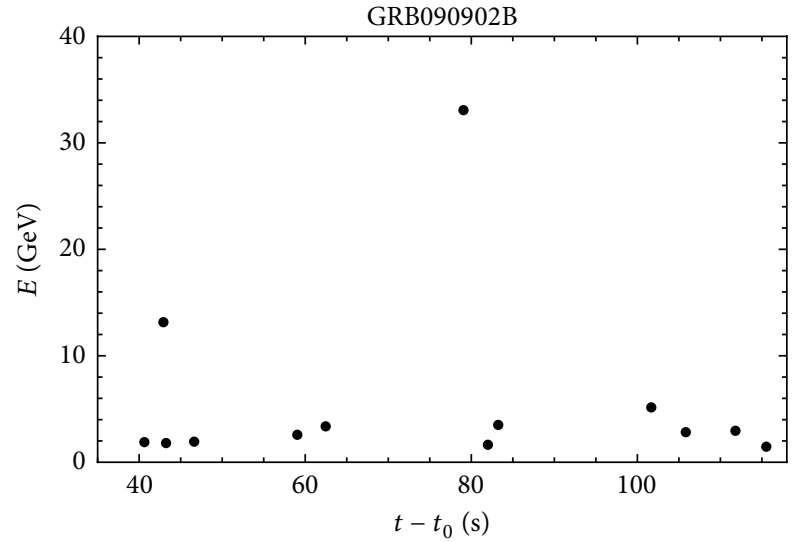

(a)

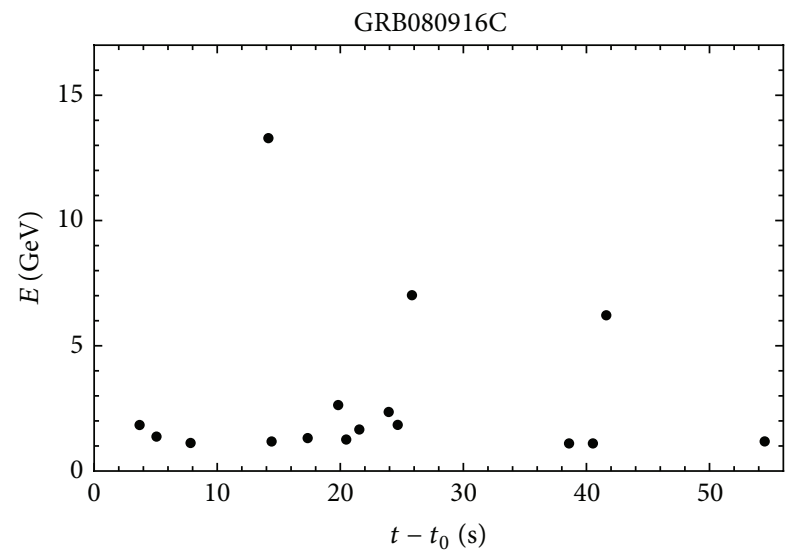

(c)

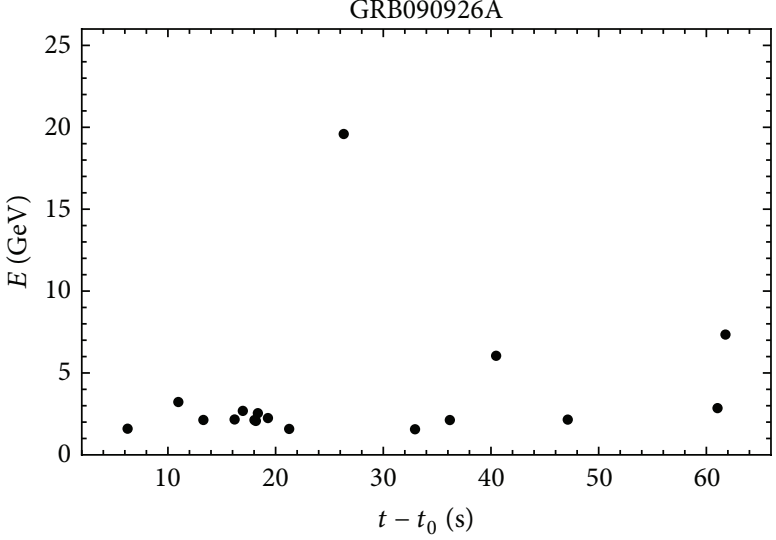

(b)

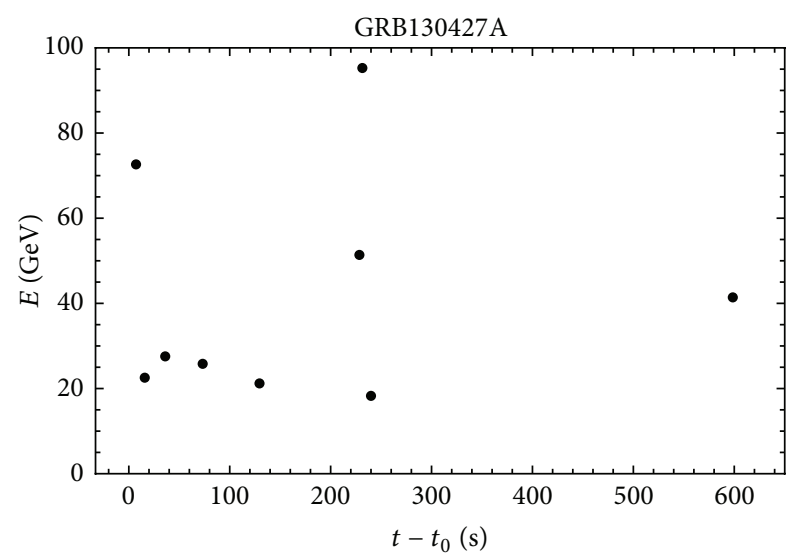

(d)

FIGURE 15: Here (d) shows data points with energy greater than $15 \mathrm{GeV}$, which illustrate the structure of our hard minibursts: a miniburst occurring between 230 and 260 seconds after trigger is preceded by a relatively long time interval ( 100 seconds) of "high-energy silence," and farther back in time one finds rather frequent detections of high-energy photons. Even for GRB130427A (observed in "high resolution") the hard minibursts are composed of only a few photons, characterized by soft spectral lags, with in particular the highest-energy photon in the miniburst observed as the first photon in the miniburst. When we looked for supporting evidence for these features within the three brightest pre-GRB130427A Fermi-LAT long GRBs (other three panels in this figure (a), (b), and (c)) we did not find conclusive evidence of small bunches of photons governed by soft spectral lags. This also suggests that the size of the spectral lags within such hard minibursts gets bigger at higher redshift. We did find that in all three of these pre-GRB130427A Fermi-LAT long GRBs, when selecting only events above a suitably-high energy, the highest-energy photon was observed after a relatively large time interval of "high-energy silence," as first photon of a phase of rather frequent detections of high-energy photons.

Let us therefore summarize the most striking elements in support of this spacetime interpretation which were encountered in our analysis. For this purpose, since we are going to compare data obtained from GRBs at different redshifts, it is useful to look at data from the perspective of the following way to rewrite (1) (restricting of course our focus to $s_{ \pm}=1$ ):

$$
E=\frac{c M_{\mathrm{QG}}}{D(0.34)} \tau_{\mathrm{QG}}(z),
$$

with

$$
\tau_{\mathrm{QG}}(z)=-\frac{D(0.34)}{D(z)} t_{\mathrm{QG}} .
$$

By rewriting (1) in this way we can place data from different GRBs (at different redshifts) on the same $E$-versus- $\tau_{\mathrm{QG}}(z)$ plots. The change of perspective we are now adopting is to compare data not by rescaling the slope on the basis of redshift but instead equivalently rescaling the measured times $t_{\mathrm{QG}}$ in such a way as to englobe the redshift dependence of $D(z)$ in the new time observable $\tau_{\mathrm{QG}}(z)=$ $-D(0.34) t_{\mathrm{QG}} / D(z)$.

This allows us to produce Figure 16 in which we collect the 7 events from GRB130427A already considered in Figure 4, the mentioned 4 events from GRB080916C that are consistent with our (1) based straight line of soft spectral lags, and we tentatively also show the two candidate GRB neutrinos of 3.3 TeV and $109 \mathrm{TeV}$ discussed in Section 4.5. The horizontal orange segments reflect a $30 \%$ energy uncertainty for the neutrinos, while the vertical segments reflect an uncertainty in the computation of $\tau_{\mathrm{QG}}(z)$ for those two neutrinos, 
which, as mentioned, is due to the fact that the redshifts of the GRBs to which they are tentatively associated is not known. Specifically the two neutrino points are for a $3.3 \mathrm{TeV}$ (with 30\% uncertainty) neutrino with $t_{\mathrm{QG}}$ of -3594 seconds assumed to have originated from a source at redshift between 0.2 and 1 (the short GRB090219) and for a $109 \mathrm{TeV}$ (with 30\% uncertainty) neutrino with $t_{\mathrm{QG}}$ of -14 hours assumed to have originated from a source at redshift between 0.3 and 6 (the long GRB091230A).

Figure 16 contains information from 13 data points, much of which was unconstrained by the setup of the analysis. We already discussed in Section 3 how the information contained in the seven points from GRB130427A is only in part used to the determine the slope of our linear relation between energy and time of detection and the parameters of rigid time translation needed to align the 3 straight lines from Figure 3. Since the slope of the straight line in Figure 16 is still the one determined using that procedure, the information in the remaining 6 points in Figure 16 was largely unconstrained within our analysis. The two neutrino points are located in the figure just where they naturally should be: for spectral lags of thousands of seconds with respect to a GRB trigger there is nothing to be gained by leaving some freedom for the actual emission time of the neutrino within the GRB event. The information in the 4 points from GRB080916C was nearly all unconstrained: the points were only used to determine the single parameter of rigid time translation needed to reset the onset of that candidate hard miniburst of GRB080916C conveniently at $\tau_{\mathrm{QG}}=0$.

The message of Figure 16 is rather intriguing and may encourage further investigation (in future studies and particularly with future observations) of the quantum-spacetime interpretation of the features we here exposed.

But as much as the message contained in Figure 16 is intriguing, we feel that on balance our findings disfavour the quantum-spacetime interpretation. A first aspect of this intuition merely originates from theory prejudice: as mentioned there are solid models of spacetime quantization that predict a behaviour of the type given in (1) but none of these known models predicts that the onset of the validity of (1) should be abrupt at some energy scale. We get the intriguing picture of Figure 16 focusing on a few high-energy photons from GRBs, but the possibility that the spectral lags predicted by the straight line in Figure 16 apply also to the abundant observations of lower-energy GRB photons is already excluded experimentally [22, 24-28]. And, as mentioned, the available literature suggests that at energies above the range which was here considered for Fermi-LAT GRBs observations one should take into account data from observations of some Blazars which also exclude the applicability of (1) with $M_{\mathrm{QG}}$ as low as $M_{\text {planck }} / 25$.

Of course we could (and eventually would) set aside this theory prejudice in the face of a clear experimental situation showing the validity of (1) only in a rather specific energy range. But even then there is a severe interpretational challenge in the data which resides in a single but very well observed photon: the $31 \mathrm{GeV}$ event in GRB090510. That $31 \mathrm{GeV}$ event occurred during a time of about 0.2 seconds for which Fermi's LAT observed a rather intense burst

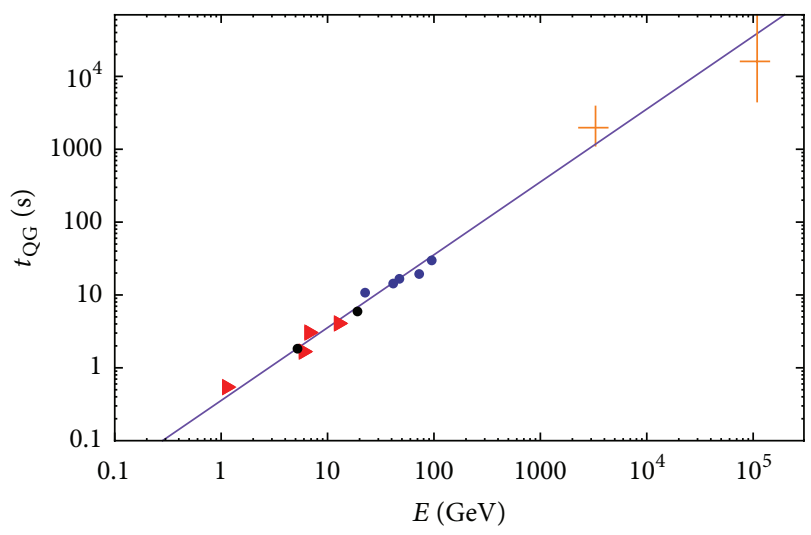

FIGURE 16: We here show in a logarithmic plot a straight line with the slope we determined in Section 3. Also shown (as thick points) are the 7 data points for GRB130427A already shown in Figure 4. The red triangles describe data on four events found on a single spectrallag straight line in Figure 10 for GRB080916C. The orange point with sizable error bars are for the $3.3 \mathrm{TeV}$ neutrino and the $109 \mathrm{TeV}$ neutrino discussed from our perspective in [32], with 30 percent uncertainty on neutrino energies reflected in horizontal segments and vertical segments reflecting the uncertainty in redshift of the GRB possibly associated to the two neutrinos.

of photons with energy between 1 and $2 \mathrm{GeV}$. Figure 14 suggests that one could interpret that $31 \mathrm{GeV}$ event consistently with (1) and consistently with the slope determined in Figure 4 from GRB130427A data by arguing that the coincidence in timing is accidental: the $31 \mathrm{GeV}$ event should have happened to occur at some point during those special 0.2 seconds just by accident, without having any connection between the mechanism and time of emission of the 1-to$2 \mathrm{GeV}$ photons being detected in that 0.2 seconds interval and the mechanism and time of emission of that $31 \mathrm{GeV}$ photon. This is not impossible but it is hard to believe.

5.3. The Possibility of an Astrophysical Interpretation. Our study was set in motion by interest in possible quantumspacetime signatures, but we stumbled upon features for which, as stressed in the previous subsection, we remain open to a quantum-spacetime interpretation (which however would require a novel formalism, not exactly producing (1), at least not at all scales) but ended up actually favouring an astrophysical interpretation, as intrinsic properties of the mechanisms producing the GRBs.

The only other case we contemplated in the previous sections as an alternative to the case of redshift dependence of the slope governed by the $D(z)$ of (1) is the case of a slope that is redshift independent and context independent. For us this served exclusively the purpose of placing in some perspective the outcome of our investigation of the hypothesis of redshift dependence governed by (1). Of course if one was to exclude the interpretation as a quantumspacetime/propagation effect, it would be most natural not to think of some redshift independent and context independent features, but rather think of intrinsic features of astrophysical origin, and with some context dependence. 
An example of avenue for a description based on intrinsic properties of the sources can be inspired by the fact that the features here discussed all are relevant at very high energies: this may suggest an inverse-compton origin, which in turn may allow a description of the soft spectral lags in terms of the corresponding cooling time being faster at progressively high energies.

We should stress that, independently of the actual nature of the mechanism involved, even within an astrophysical interpretation of the timing features we here highlighted one could expect some redshift dependence. To see this one can think of the simple-minded hypothesis of an energydependent timing features which at the emission are always exactly the same: then because of redshift and time dilation one would have a picture of such energy-dependent timing features at detection which does depend on redshift. Such an interpretation would lead one to contemplate for our picture the possibility that the slope of the relevant linear dependence might scale with redshift according to $(1+z)^{-2}$. So in comparing this slope of linear spectral lags between a GRB at redshift of $z_{1}$ and a redshift of $z_{2}$ one would expect a behaviour at least roughly rescaling the slope by $\left(1+z_{1}\right)^{2} /(1+$ $\left.z_{2}\right)^{2}$ (given a certain value for the slope found for a GRB at redshift $z_{1}$ then for a GRB at higher redshift $z_{2}$ one would expect the slope to be less steep by a factor of $\left(1+z_{1}\right)^{2} /(1+$ $\left.z_{2}\right)^{2}$ ).

It is interesting to observe that this natural estimate of $\left(1+z_{1}\right)^{2} /\left(1+z_{2}\right)^{2}$ for the redshift dependence in case of an astrophysical interpretation leads to a quantification which is not wildly different from the quantum-spacetime prediction $D\left(z_{1}\right) / D\left(z_{2}\right)$. We give an intuitive characterization of this in Figure 17, where we compare the two predictions for the case of GRB080916C, which is the highest-redshift GRB among the bright GRBs we here considered.

Confirming what we just stressed, the blue and the red lines in Figure 17 reflect slopes which are not wildly different and as a result they lead to a comparably compelling picture based on the hypothesis of linear soft spectral lags for the data available for GRB080916C. This also shows that (assuming that linear soft spectral lags within hard miniburst are eventually fully established) it might require more than just a few GRBs with suitable properties in order to distinguish between the hypothesis of slope with astrophysics-inspired redshift dependence of $\left(1+z_{1}\right)^{2} /\left(1+z_{2}\right)^{2}$ and the hypothesis of slope with the quantum-spacetime-inspired redshift dependence of $D\left(z_{1}\right) / D\left(z_{2}\right)$.

5.4. Outlook. We here provided evidence both for the more general structure of hard minibursts, as perhaps best summarized in Figure 15, and for the specific feature of linearin-energy soft spectral lags within the development of such hard minibursts, as perhaps best summarized in Figures 5, 7, and 16.

In gaining an intuition for the robustness of the more general hard-miniburst structure one should take into account on one side the fact that Figure 15 involves all the FermiLAT long GRBs bright enough for one to search for such a

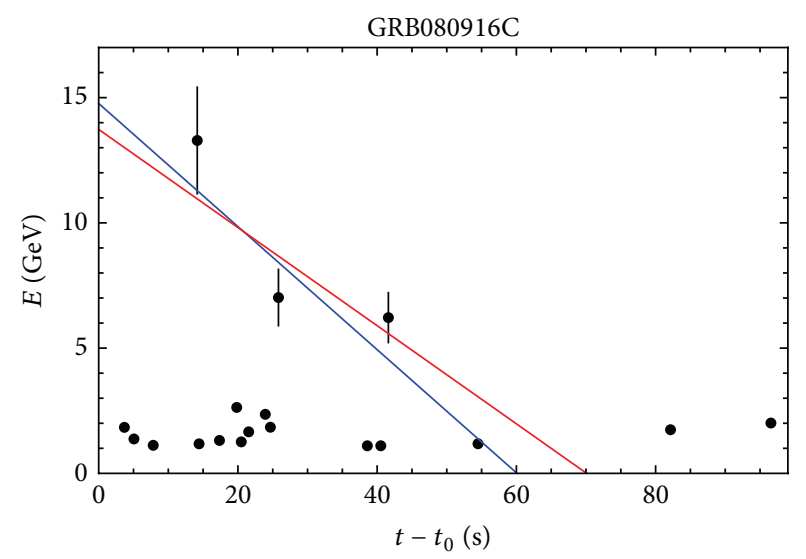

FIGURE 17: The blue line and the data points in this figure exactly match the ones for Figure 10, concerning GRB08091C. The only difference is that now we add the red line whose slope is obtained from the slope fit in Figure 4 (for GRB130427A) by rigidly rescaling it by the factor of $1.34^{2} / 5.35^{2}$, that is, $\left(1+z_{1}\right)^{2} /\left(1+z_{2}\right)^{2}$ for $z_{1}=0.34$ (GRB130427A) and $z_{2}=4.35$ (GRB080916C).

feature and on the other side the fact that these are after all just 4 GRBs. While the "lower resolution" of observed highenergy images of more distant GRBs did not allow us to conclusively identify bunches of photons governed by soft spectral lags, we did interpret as encouraging the consistent pattern highlighted in Figure 15 for the structure of a time interval around the time of detection of the highest-energy photon in a GRB. Also considering that this hard-miniburst feature is something that was not expected by us and was recognized on available data (rather than being predicted in advance) we feel it should at this point be considered as a promising hypothesis to be tested on future data. In this respect we should acknowledge that a limitation of the notion of hard miniburst which we here introduced is its rather qualitative nature, indeed best summarized in Figure 15. We notice however that a quantitative requirement could be based on the studies here reported in Section 3.3. There we focused on "late times", essentially defined as times starting with the detection of the highest-energy photon in the burst, and we used Monte Carlo-randomization criteria for showing that in that late-time window the data on GRB130427A manifest a strong bias toward soft spectral lags that is rather unlikely as just a chance occurrence. This is a quantitative aspect of our hypothesis that the highest-energy photons in a long GRB might all be part of hard miniburst involving soft spectral lags (as here observed for GRB130427A).

For what concerns the robustness of the hypothesis that at least in some bright high-energy GRBs such hard minibursts involve soft spectral lags with linear dependence on energy the most impressive indication of significance of the evidence we here found is contained in Figure 5 and in Figure 7. Especially Figure 7 indicates that this feature is present with a rather high statistical significance in GRB130427A. Still some prudence may be invited by the observation that this statistical significance emerges from a remarkable sharp timing feature for just very few events (think in particular 
of the blue line in Figure 7, which focuses only on the few events with energy greater than $15 \mathrm{GeV}$ at times greater than 190 seconds after the GRB130427A trigger). This of course is automatically taken into account in the assessment of the statistical significance shown in Figure 7, but still somehow, because of the fact that only few events are involved, we do feel it would be important to gather additional evidence. This aspect however illustrates very clearly the significance of the observation of GRB130427A for the overall effort of understanding GRBs: one could perhaps have noted the implications for linear soft spectral lags of Figure 10 for GRB080916C, but that observation, even when combined with a few similar observations for other bright GRBs, could never lead to a robust indication. The indication in favour of linear soft spectral lags contained in the "high-resolution" image for GRB130427A contained in Figure 3 brings us already so close to conclusive evidence that we can already describe Figure 10 for GRB080916C as supporting evidence. In order for the evidence to be assessed as conclusive, it would now take only a few more GRBs, even with "low-resolution" images like Figure 10 for GRB080916C.

Even taking as working assumption that this linear soft spectral lags are a true feature a lot remains to be worked out about their interpretation. Evidently the most fascinating interpretation would be the one based on quantum-spacetime effects. This quantum-spacetime interpretation requires that the effects are present in exactly the same way in all bursts, with the only differences allowed being governed by redshift, through a function of redshift of the type of the $D(z)$ of (1). One is allowed (in spite of this being something that lacks any support in the quantum-spacetime formalisms so far explored) to speculate about possible scales of abrupt onset or offset of the spacetime effects, but a dependence on redshift of the type of the $D(z)$ is nonnegotiable for the quantum-spacetime interpretation. At least on the basis of the consistency between Figure 3 for GRB130427A and Figure 10 for GRB080916C further exploration of this interpretation is to some extent encouraged. But the specific model based on (1), extending over all values of energy, is clearly inadequate for such explorations because of the limits established for effects of this magnitude in previous studies of GRB photons at energies below the ones that played a key role here and in previous studies of blazars at energies above the ones that played a key role here. Moreover, as we also stressed, the quantum-spacetime interpretation of the features here exposed is significantly challenged by the applicability to GRB090510, with its $31 \mathrm{GeV}$ photon detected in sharp time coincidence with several photons of energy between $1 \mathrm{GeV}$ and $2 \mathrm{GeV}$.

Considering the outmost importance of the scientific issues at stake the quantum-spacetime avenue should be nonetheless pursued vigorously, but our findings evidently favour a description of the features here highlighted based on intrinsic properties of the sources, in terms of a pure astrophysics interpretation.

It is intriguing that even if spectral lags within our hard minibursts do not have a quantum-spacetime origin their understanding will be important for quantum-spacetime research: the search of possibly minute quantum-spacetime- induced spectral lags evidently requires as a prerequisite our best possible understanding of intrinsic mechanisms at the sources that can produce spectral lags. As the understanding of our hard minibursts improves they may well turn into "standard candles," with distinctive standard features, ideally suited for the search of possible additional contributions to the spectral lags with characteristic dependence on redshift of the type $D(z)$ and therefore attributable to quantumspacetime effects.

\section{Conflict of Interests}

The authors declare that there is no conflict of interests regarding the publication of this paper.

\section{Acknowledgment}

This research was supported in part by the John Templeton Foundation (GAC).

\section{References}

[1] S. Zhu, J. Racusin, D. Kocevski et al., "Fermi-LAT detection of a burst,” GCN Circular, no. 14471, GRB 130427A, 2013.

[2] A. von Kienli, "Fermi GBM observation," GCN Circular, no. 14473, GRB 130427A, 2013.

[3] A. Maselli, A. P. Beardmore, A. Y. Lien et al., "Swift detection of a very bright burst with a likely bright optical counterpart," GCN Circular, no. 14448, GRB 130427A, 2013.

[4] S. Golenetskii, R. Aptekar, D. Frederiks et al., "Konus-Wind observation of GRB 130427A," GCN Circulars, no. 14487, 2013.

[5] A. Pozanenko, P. Minaev, and A. Volnova, "SPIACS/INTEGRAL observations," GCN Circulars, no. 14484, GRB 130427A, 2013.

[6] F. Verrecchia, C. Pittori, A. Giuliani et al., "High energy gammaray detection by AGILE," GCN Circulars, no. 14515, GRB 130427A, 2013

[7] A. Chernenko and I. Mitrofanov, "Decomposition of a cosmic gamma-ray burst into two non-correlated radiation components," Monthly Notices of the Royal Astronomical Society, vol. 274, no. 2, pp. 361-368, 1995.

[8] H. Flores, S. Covino, D. Xu et al., "VLT/X-shooter redshift confirmation," GCN Circulars, no. 14491, GRB 130427A, 2013.

[9] A. J. Levan, S. B. Cenko, D. A. Perley, and N. R. Tanvir, "GeminiNorth redshift," GCN Circulars, no. 14455, GRB 130427A, 2013.

[10] D. Xu, C. Cao, S.-M. Hu, and C.-M. Zhang, "Weihai optical observations," GCN Circulars, no. 14458, GRB 130427A, 2013.

[11] L. Elenin, A. Volnova, V. Savanevych, A. Bryukhovetskiy, I. Molotov, and A. Pozanenko, "Early optical observations," GCN Circulars, no. 14450, GRB 130427A, 2013.

[12] M. Ackermann, M. Ajello, L. Baldini et al., "Constraints on the cosmic-ray density gradient beyond the solar circle from Fermi $\gamma$-ray observations of the third galactic quadrant," The Astrophysical Journal, vol. 726, no. 2, article 81, 2011.

[13] W. B. Atwood, A. A. Abdo, M. Ackermann et al., "The large area telescope on the Fermi gamma-ray space telescope mission," The Astrophysical Journal, vol. 697, no. 2, Article ID 1071, 2009.

[14] M. Ackermann, M. Ajello, A. Albert et al., "The Fermi large area telescope on orbit: event classification, instrument response 
functions, and calibration," The Astrophysical Journal Supplement Series, vol. 203, no. 1, article 4, 2012.

[15] G. Amelino-Camelia, J. Ellis, N. E. Mavromatos, D. V. Nanopoulos, and S. Sarkar, "Tests of quantum gravity from observations of $\gamma$-ray bursts," Nature, vol. 393, pp. 763-765, 1998.

[16] R. Gambini and J. Pullin, "Nonstandard optics from quantum space-time," Physical Review D, vol. 59, no. 12, Article ID 124021, 4 pages, 1999.

[17] J. Alfaro, H. A. Morales-Tecotl, and L. F. Urrutia, "Quantum gravity corrections to neutrino propagation," Physical Review Letters, vol. 84, no. 11, pp. 2318-2321, 2000.

[18] G. Amelino-Camelia and S. Majid, "Waves on noncommutative space-time and gamma-ray bursts," International Journal of Modern Physics A, vol. 15, no. 27, article 4301, 2000.

[19] R. C. Myers and M. Pospelov, "Ultraviolet modifications of dispersion relations in effective field theory," Physical Review Letters, vol. 90, no. 21, Article ID 211601, 4 pages, 2003.

[20] U. Jacob and T. Piran, "Lorentz-violation-induced arrival delays of cosmological particles," Journal of Cosmology and Astroparticle Physics, vol. 2008, no. 1, article 031, 2008.

[21] D. Mattingly, “Modern tests of Lorentz invariance," Living Reviews in Relativity, vol. 8, article 5, 2005.

[22] G. Amelino-Camelia and L. Smolin, "Prospects for constraining quantum gravity dispersion with near term observations," Physical Review D, vol. 80, no. 8, Article ID 084017, 14 pages, 2009.

[23] R. J. Szabo, "Quantum field theory on noncommutative spaces," Physics Reports, vol. 378, no. 4, pp. 207-299, 2003.

[24] A. A. Abdo, M. Ackermann, M. Ajello et al., "A limit on the variation of the speed of light arising from quantum gravity effects," Nature, vol. 462, pp. 331-334, 2009.

[25] A. Abdo, M. Ackermann, M. Ajello et al., "Detection of high-energy gamma-ray emission from the globular cluster 47 Tucanae with Fermi," Science, vol. 323, no. 5942, pp. 845-848, 2009.

[26] J. Ellis, N. E. Mavromatos, and D. V. Nanopoulos, "Probing a possible vacuum refractive index with $\gamma$-ray telescopes," Physics Letters B, vol. 674, no. 2, pp. 83-86, 2009.

[27] J. Bolmont and A. Jacholkowska, "Lorentz symmetry breaking studies with photons from astrophysical observations," Advances in Space Research, vol. 47, no. 2, pp. 380-391, 2011.

[28] R. J. Nemiroff, R. Connolly, J. Holmes, and A. B. Kostinski, "Bounds on spectral dispersion from Fermi-detected gamma ray bursts," Physical Review Letters, vol. 108, no. 23, Article ID 231103, 4 pages, 2012.

[29] J. Albert, E. Aliu, H. Anderhub et al., "Probing quantum gravity using photons from a flare of the active galactic nucleus Markarian 501 observed by the MAGIC telescope," Physics Letters B, vol. 668, no. 4, pp. 253-257, 2008.

[30] F. Aharonian, A. G. Akhperjanian, U. B. de Almeida et al., "Limits on an energy dependence of the speed of light from a flare of the active galaxy PKS 2155-304," Physical Review Letters, vol. 101, no. 17, Article ID 170402, 5 pages, 2008.

[31] M. Ackermann, M. Ajello, K. Asano et al., "The first Fermi-LAT gamma-ray burst catalog," The Astrophysical Journal Supplement Series, vol. 209, no. 1, article 11, 2013.

[32] G. Amelino-Camelia, D. Guetta, and T. Piran, "Possible relevance of quantum spacetime for neutrino-telescope data analyses," http://arxiv.org/abs/1303.1826.

[33] R. Abbasi, Y. Abdou, T. Abu-Zayyad et al., "An absence of neutrinos associated with cosmic-ray acceleration in $\gamma$-ray bursts," Nature, vol. 484, pp. 351-354, 2012.
[34] N. Whitehorne, A search for high-energy neutrino emission from gamma-ray bursts [Ph.D. thesis], University of WisconsinMadison, Madison, Wis, USA, 2012, https://docushare .icecube.wisc.edu/dsweb/Get/Document-60879/thesis.pdf.

[35] D. Wanderman and T. Piran, "The luminosity function and the rate of Swift's gamma-ray bursts," Monthly Notices of the Royal Astronomical Society, vol. 406, no. 3, pp. 1944-1958, 2010.

[36] D. Coward, E. J. Howell, T. Piran et al., "The Swift short gammaray burst rate density: implications for binary neutron star merger rates," Monthly Notices of the Royal Astronomical Society, vol. 425, no. 4, pp. 2668-2673, 2012. 

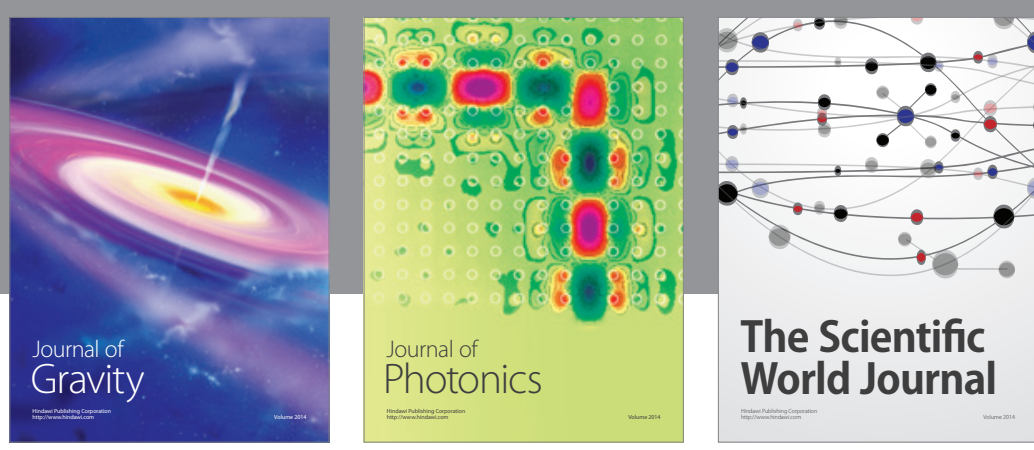

The Scientific World Journal
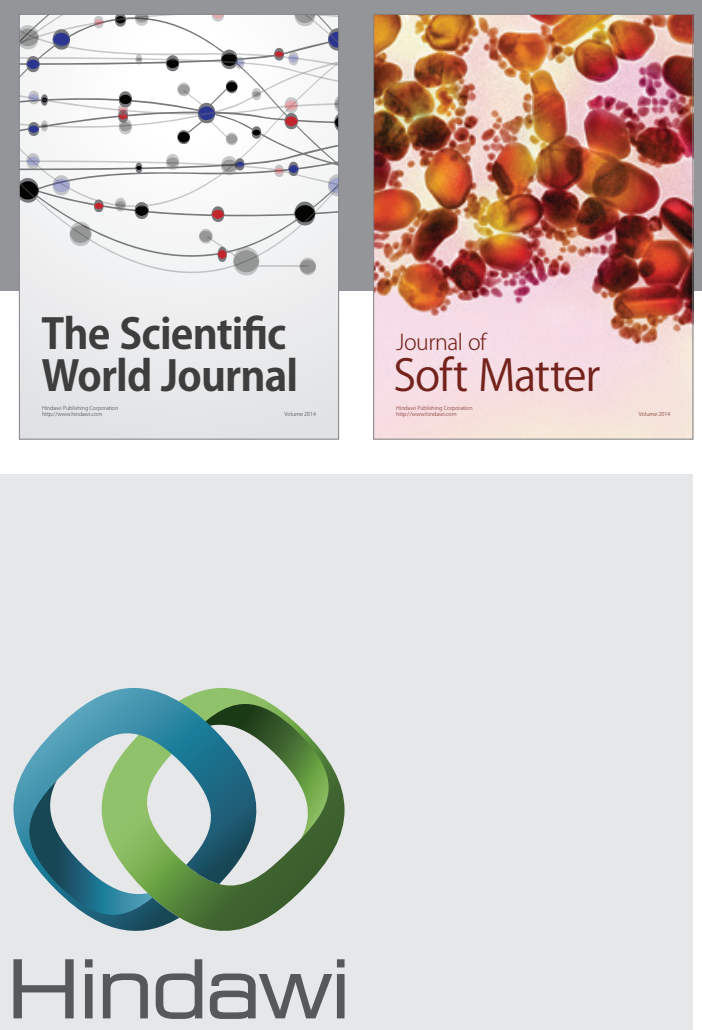

Submit your manuscripts at

http://www.hindawi.com

nternational Journal of

Statistical Mechanics
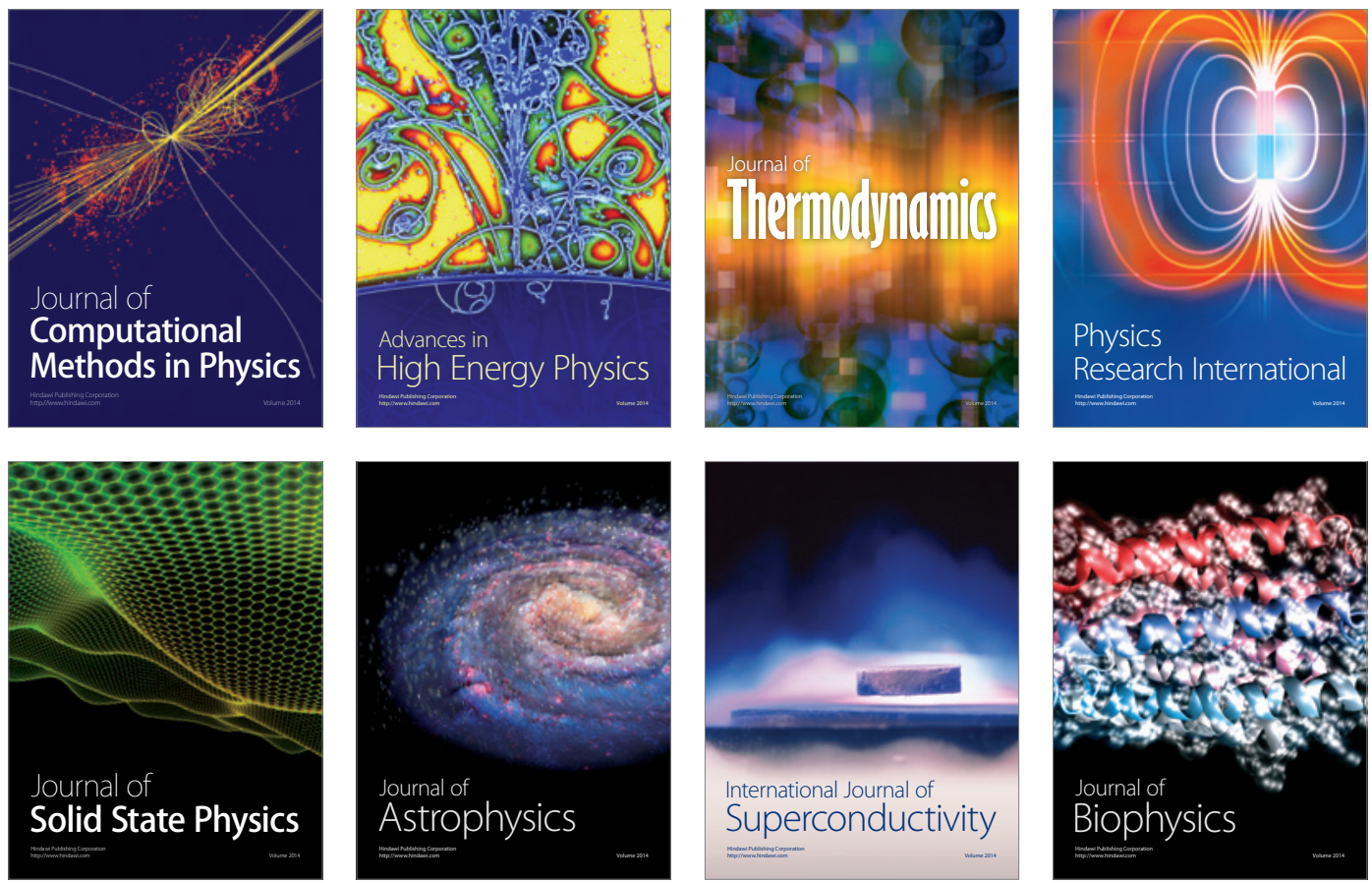
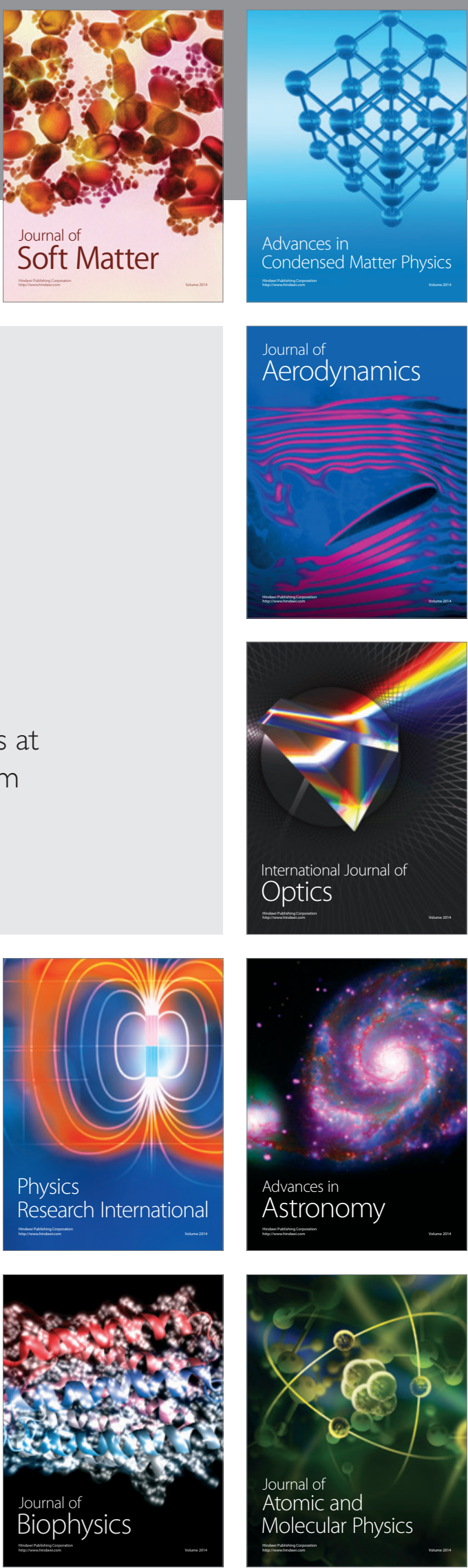